\title{
Loss of function of DJ-1 triggered by Parkinson's disease-associated mutation is due to proteolytic resistance to caspase- 6
}

\author{
E Giaime ${ }^{1}$, C Sunyach ${ }^{1}$, C Druon ${ }^{1}$, S Scarzello ${ }^{1}$, G Robert ${ }^{2}$, S Grosso ${ }^{2}$, P Auberger ${ }^{2}$, MS Goldberg ${ }^{3,6}$, J Shen ${ }^{3}$, P Heutink ${ }^{4}$, \\ $\mathrm{J}$ Pouysségur ${ }^{5}$, G Pagès ${ }^{5}, \mathrm{~F}$ Checler $^{*, 1}$ and $\mathrm{C}$ Alves da Costa ${ }^{*, 1}$
}

DJ-1 was recently identified as a gene product responsible for a subset of familial Parkinson's disease (PD). The mechanisms by which mutations in DJ-1 alter its function and account for PD-related pathology remained largely unknown. We show that DJ-1 is processed by caspase- 6 and that the caspase-6-derived C-terminal fragment of DJ-1 fully accounts for associated p53dependent cell death. In line with the above data, we show that a recently described early-onset PD-associated mutation (D149A) renders DJ-1 resistant to caspase-6 proteolysis and abolishes its protective phenotype. Unlike the D149A mutation, the L166P mutation that prevents DJ-1 dimerization does not impair its proteolysis by caspase- 6 although it also abolishes DJ-1 antiapoptotic function. Therefore, we show here that DJ- 1 loss of function could be due to impaired caspase- 6 proteolysis and we document the fact that various DJ-1 mutations could lead to PD pathology through distinct molecular mechanisms.

Cell Death and Differentiation (2010) 17, 158-169; doi:10.1038/cdd.2009.116; published online 14 August 2009

Parkinson's disease (PD) is a movement disorder, the incidence of which increases sharply with age. It is characterized by a massive loss of dopaminergic neurons of the substantia nigra pars compacta and the presence of intracytoplasmic inclusions named Lewy bodies. Most of PD cases are of sporadic origin but about five percent of them are of genetic origin and are either associated to an autosomaldominant or recessive mode of transmission. The latter forms of the disease are usually associated to an early onset $(<50$ years old) and are linked to mutations in the genes of parkin, PINK-1 and DJ-1. ${ }^{1}$

DJ-1 is implicated in approximately $1-2 \%$ of recessive forms of $\mathrm{PD}^{2,3}$ and is a ubiquitous highly conserved protein that is normally expressed in the brain as a homodimeric complex. ${ }^{4}$ To our knowledge, to date, only two DJ-1 mutations corresponding to a deletion of exons $1-5$ and a point mutation that converts the leucine residue in position 166 into a proline (referred to as L166P-DJ-1 hereafter), have been identified in a Dutch and an Italian family, respectively. That these DJ-1 mutations triggered a drastic decrease in DJ-1 levels suggested that these familial cases were likely because of a loss of stability of DJ-1. In apparent contradiction with these conclusions, recently, a heterozygous DJ-1 mutation (D149A) was reported to lead to early-onset PD whereas DJ-1 appeared catabolically stabilized. ${ }^{2,5}$

Little is known about the physiological function of DJ-1 and the mechanisms by which DJ-1 mutations lead to PD although invalidation of DJ-1 clearly established dopaminergic deficits and hypokinesia. ${ }^{6} \mathrm{~A}$ few functional studies indicated that as a member of the ThiJ/Pfpl family, DJ-1 could act as a molecular chaperone. $^{7}$ It has also been suggested that DJ-1 may possess RNA-binding properties ${ }^{8}$ and may lead to transcriptional activation through the interaction with $\mathrm{PIASx}^{9}$ that is a ubiquitin ligase involved in the process of sumoylation of several proteins. ${ }^{10}$ It should be noted that DJ-1 itself is sumoylated, suggesting a role of this protein in cell signaling. ${ }^{11}$ Several lines of evidence also indicate that DJ-1 may act as an oxidative stress sensor. Thus, it has been demonstrated that the cysteine 106 of $D J-1$ is essential for its acidic PI shift in oxidative stress conditions. ${ }^{12,13}$ Of most interest, the antioxidant properties of DJ-1 are associated with its ability to trigger neuroprotection. ${ }^{14}$

\footnotetext{
${ }^{1}$ Institut de Neuromédecine Moléculaire IN2M and Institut de Pharmacologie Moléculaire et Cellulaire, UMR6097 CNRS/UNSA, Équipe labellisée Fondation pour la Recherche Médicale, 660 Route des Lucioles, 06560, Sophia-Antipolis, Valbonne, France; ${ }^{2}$ Faculté de Médecine, INSERM U895, Équipe labellisée Ligue Nationale contre le Cancer, Centre Méditerranéen de Médecine Moléculaire (C3M), Team 2, Avenue de Valombrose, 06107 Nice, France; ${ }^{3}$ Center for Neurologic Diseases, Brigham \& Women's Hospital, Program in Neuroscience, Harvard Medical School, Boston, MA 02115, USA; ${ }^{4}$ Section Medical Genomics, Department of Clinical Genetics, VU University Medical Center, Amsterdam, The Netherlands and ${ }^{5}$ Institute of Signaling, Developmental Biology and Cancer Research, CNRS UMR 6543, Centre A. Lacassagne, 33 Avenue de Valombrose, 06189 Nice, France

*Corresponding authors: F Checler and C Alves da Costa, Institut de Neuromédecine Moléculaire IN2M and Institut de Pharmacologie Moléculaire et Cellulaire, UMR6097 CNRS/UNSA, Équipe labellisée Fondation pour la Recherche Médicale, 660 Route des Lucioles, 06560, Sophia-Antipolis, Valbonne, France. Tel: 3349395 7759 or 3349395 7760; Fax: 3349395 7708; E-mails: acosta@ipmc.cnrs.fr or checler@ipmc.cnrs.fr

${ }^{6}$ Present address: Departments of Neurology and Psychiatry, UT Southwestern Medical Center, 5323 Harry Hines Blvd., Dallas, TX 75390 USA

Keywords: Parkinson's diseased; DJ1; p53; caspase 6; autosomal recessive cases; mutations

Abbreviations: PD, Parkinson's disease; STS, staurosporine; 6OHDA, 6-hydroxydopamine; Ac-VEID-al, N-Acetyl-Val-Glu-Ile-Asp-al; Ac-DEVD-7AMC, N-Acetyl-AspGlu-Val-Asp-7-amino-4-methylcoumarin; ALLN, N-acetyl-L-leucyl-L-norleucinal; AEBSF, [4-(2-aminoethyl) benzenesulphonyl fluoride hydrochloride]; E-64, [transepoxysuccinyl-L-leucylamido-(4-guanidino)butane]; TSM1, Telencephalon-specific mouse 1; HEK293, human embryonic kidney 293; MEF, mouse embryonic fibroblasts; PAGE, polyacrylamide gel electrophoresis; HEPES, (4-(2-hydroxyethyl)piperazine-1-ethanesulfonic acid) buffer; CHAPS, ((3-[(3-cholamidopropyl)dimethylamonio]-1-propanesulfonate)); PCR, polymerase chain reaction; ALS, Amyotrophic lateral sclerosis; PARP, Poly-ADP-ribose polymerase

Received 16.1.09; revised 04.6.09; accepted 06.7.09; Edited by D Kaplan; published online 14.8.09
} 
The mechanisms by which DJ-1 elicits neuroprotection are far from being elucidated. Here, we show that the overexpression of DJ-1 in neuronal and dopaminergic cells elicits a p53-dependent protective response against various PD and non-PD-associated stimuli. Thus, cells overexpressing wildtype DJ-1 display decreased p53 expression, promoter transactivation and mRNA levels by an Akt-dependent signaling whereas conversely, DJ-1 depletion triggers an upregulation of the p53 pathway in both cellular and knockout animal models. Functional comparison studies between L166P-DJ-1 and D149A-DJ-1 indicate that both mutations abolish DJ-1-associated control of p53. However, L166P-DJ1 and D149A-DJ-1 display distinct susceptibility to caspase-6. Thus, unlike L166P, the D149A mutation fully blocks DJ-1 cleavage by recombinant caspase- 6 . This proteolytic resistance fully explains the loss of function of DJ-1 because we show that the C-terminal fragment of $\mathrm{DJ}-1$ derived from its cleavage by caspase- 6 totally accounts for the DJ-1-mediated protective function. Interestingly, we show that the levels of DJ-1 and caspase- 6 are inversely correlated in human brain samples derived from sporadic PD patients, suggesting a role of caspase- 6 in the physiological control of DJ-1 brain levels and its loss of function in the pathology. Overall, this is the first demonstration of the selective implication of caspase- 6 in the proteolysis of DJ-1 and that the blockade of this process is likely responsible for a subset of autosomal recessive early onset PD cases.

\section{Results}

PD-associated mutations abolish the protective phenotype of DJ-1 in mouse and human neuronal cells. We have obtained stable transfectants overexpressing wild-type DJ-1 (WT-DJ), D149A-DJ-1 (149DJ) and L166P-DJ-1 (166DJ) in TSM1 neurons. The WTDJ and 149DJ clones chosen for experiments express similar DJ-1 protein (Figure 1a, upper) and mRNA levels (Figure 1a, lower). Although similar mRNA levels were observed for 166DJ-1 (Figure 1a lower) reflecting equivalent transfection efficiencies, only longer exposure of SDS-polyacrylamide gel electrophoresis (PAGE) analysis allowed to visualize the V5-tagged protein, confirming the previously reported catabolic instability of L166P-DJ-1. ${ }^{15}$ To
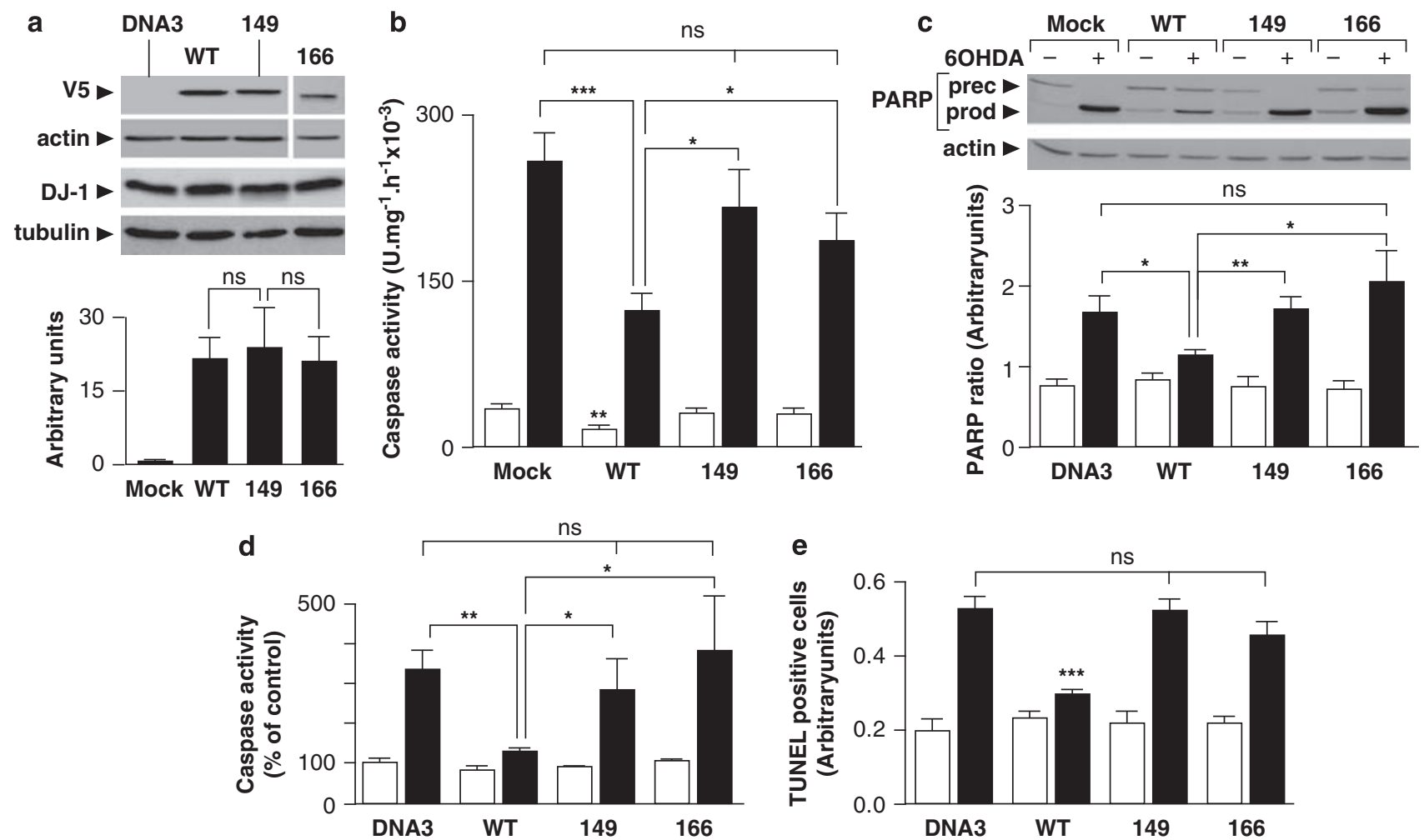

Figure 1 DJ-1 lowers staurosporine-induced caspase-3 activation in human and murine neuronal cells: effect of PD-associated mutations. (a) TSM1 cells were stably transfected with the indicated cDNA then V5-tagged DJ-1, endogenous DJ-1 and actin expressions (a, upper panel) and mRNA levels (a, lower panel) were analyzed by western blot and real-time PCR, respectively as described in the 'Materials and Methods'. Bars correspond to mRNA levels expressed in arbitrary units and are the means \pm S.E.M of three independent experiments performed in triplicates. (b, c) The indicated TSM1 cell lines were treated for $8 \mathrm{~h}$ with vehicle (empty bars) or with $60 \mathrm{OHDA}$ $(0.2 \mathrm{mM}$, black bars) then caspase-3 activity (b) and poly-ADP-ribose polymerase cleavage (c) were analyzed as described in the 'Materials and Methods'. Bars in $\mathbf{b}$ and $\mathbf{c}$ are the means \pm S.E.M of 10 and three independent experiments performed in duplicates, respectively. ${ }^{\star} P<0.05 ;{ }^{* \star *} P<0.001$, ns, not statistically significant. PARP ratio corresponds to PARP product over PARP precursor expressions. (d, e) SH-SY5Y human neuroblastoma cells were transiently transfected with the indicated cDNAs. Twentyfour hours after transfection, cells were treated for $8 \mathrm{~h}$ without (empty bars) or with 6OHDA (0.2 mM, black bars) then caspase-3 activity (d) and Tunel-positive cells (e) were monitored as described in the 'Materials and Methods'. Bars in $\mathbf{d}$ correspond to caspase-3 activity expressed as percent of control (untreated mock-transfected cells) and are the means \pm S.E.M of 4-5 independent experiments performed in duplicates. ${ }^{\star} P<0.05 ;{ }^{* \star} P<0.01 ;{ }^{* \star \star} P<0,001$, ns, not statistically significant. Bars in $\mathrm{E}$ correspond to tunelpositive cells and are the means \pm S.E.M. of 8-10 independent fields 
evaluate the ability of DJ-1 and its mutated congeners to control cell death, we have challenged the cells with 6hydroxydopamine (6OHDA), a natural toxin frequently used to mimic PD in cellular and animal models. ${ }^{16}$ Figure $1 \mathrm{~b}$ shows that 6OHDA strongly activates caspase-3 in mocktransfected neurons. Clearly, WT-DJ-1 drastically reduces 6OHDA-stimulated caspase-3 activation whereas L166P and D149A mutations abrogate this phenotype. These results were further confirmed by the analysis of the cleavage of poly-ADP-ribose polymerase (PARP), a physiological substrate of caspase- 3 that is cleaved and inactivated during apoptosis. ${ }^{17}$ As expected, 6OHDA lowers PARP precursor expression and increases its proteolytic product in mock-transfected cells (Figure 1c). The quantification analysis (Figure 1c) indicates that WT-DJ-1 reduces PARP cleavage of $40 \%$ and thus confirms that WT-DJ-1 but not its mutants protects TSM1 cells from 6OHDA-induced caspase3 activation. This phenotype is not cell-specific. Thus, in human SH-SY5Y neuroblastoma cells, which are considered as one of the most relevant dopaminergic cell model, the overexpression of WT-DJ-1 reduces cellular responsiveness to 6OHDA (Figure 1d) and staurosporine (STS) (not shown) whereas both DJ-1 mutations abolished this phenotype (Figure 1d). That DJ-1-associated reduction of caspase-3 activation indeed reflected decreased cell death was confirmed by TUNEL analysis in SH-SY5Y cells (Figure 1e). Thus, overexpression of WT-DJ-1 triggers a drastic decrease of DNA fragmentation. Here again, DJ-1 mutants did not modify 6OHDA-associated cell death (Figure 1e).

\footnotetext{
Wild-type but not mutated DJ-1 downregulates p53 at both transcriptional and post-transcriptional levels. We have analyzed the potential of WT-DJ-1 and its mutants as modulators of the p53 pathway. By means of a construct in which the consensus site targeted by $p 53$ is in frame with the luciferase reporter gene (PG13), we show that WT-DJ-1 reduces by about $50 \%$ the transcriptional activity of p53 whereas both D149A and L166P mutations abolished this phenotype (PG13, Figure 2a, left panel). Accordingly, WTDJ-1 but not mutated DJ-1 decreases p53 promoter transactivation (mpp53, Figure 2a, left panel) and mRNA levels (Figure $2 \mathrm{a}$, right panel). To establish whether the antiapoptotic phenotype of DJ-1 was fully dependent of p53, we took advantage of two cell models in which either $p 19^{\text {arf }}$ or $p 19^{\text {arf }}$ and $p 53$ genes had been invalidated. The depletion of $p 19^{\text {arf }}$ avoids cell senescence and allows the analysis of the function of p53 in the control of cell death but not in that of cell cycle. ${ }^{18}$ First, it must be noted that both $\mathrm{p} 19^{\text {arf- }- \text { - }}$ and p19 arf-/- p53 $^{-/-}$fibroblasts respond to STS and 6OHDA by an activation of their endogenous caspase- 3 although to a lesser extent in the latter cell system (Figure 2b, compare DNA lanes in histograms). Transient transfection of WT-DJ-1 CDNA in the $p 19^{\text {arf }}$ cell line reduces STS (gray bars) and 6OHDA (black bars)-stimulated caspase-3 activation by about 40 and $60 \%$, respectively (Figure $2 \mathrm{~b}$, left histogram) whereas the D149A and L166P DJ-1 mutations abolished this phenotype. Interestingly, WT-DJ-1-associated reduction of caspase- 3 activation appears totally prevented by p53
}

deficiency (Figure 2b, right histogram), indicating that DJ-1 protective function is fully dependent of p53.

We examined the mechanisms by which DJ-1 could control p53. Several putative upstream p53 modulators including the PI3 kinase/Akt and extracellular-regulated kinases have been described. Thus, the Akt survival pathway was shown to downregulate $\mathrm{p} 53^{19,20}$ by triggering its Mdm2-mediated ubiquitination and subsequent proteasomal degradation ${ }^{21,22}$ whereas ERK belongs to the mitogen-activated protein kinase superfamily that targets $\mathrm{p} 53 .{ }^{23}$ Figure $3 a$ (histogram) shows that the Akt inhibitor LY294002 fully impairs the DJ-1-associated protection against 6OHDA in SH-SY5Y neuroblastoma cells. As expected, LY294002 triggers an augmentation of p53 activity, the extent of which was fully abolished by DJ-1. (Figure 3b). Conversely, ERK-1 deficiency does not affect the DJ-1-associated phenotype in fibroblasts (Figure 3c). This data clearly suggests that the Akt but not the ERK-survival pathway was mainly involved in the DJ-1associated protective function. Two lines of data led us to examine whether NF- $\kappa \mathrm{B}$ could mediate DJ-1-associated Aktdependent regulation of p53. First, two recent studies indicated that there could exist an Akt-dependent phosphorylation of the $I_{\kappa} \mathrm{B}-\mathrm{kinase}$ that ultimately led to $\mathrm{NF}-\kappa \mathrm{B}$ activation. $^{24,25}$ Second, it was recently shown that several cell systems inhibit p53 activity and enhance cell survival by an Akt-dependent activation of $\mathrm{NF}-\kappa \mathrm{B}^{26}$ In this context, we examined whether the pharmacological targeting of the $\mathrm{NF}-\kappa \mathrm{B}$ pathway could influence DJ-1-associated function. Supplementary Figure 1 shows that the $I_{\kappa} \mathrm{B}$-kinase inhibitor BMS-345541 does not affect the DJ-1-associated reduction of 6OHDA-induced caspase-3 activation. Overall, the above data show that DJ-1 modulates p53 by an Akt-dependent but ERK- and NF $\kappa$ B-independent pathway.

That DJ-1 protective function was totally blocked by LY294002 clearly suggested a fully post-transcriptional control of $p 53$ by Akt but was, at first sight, difficult to reconcile with our observation that DJ-1 also downregulated p53 promoter transactivation. One explanation could be that the post-transcriptional Akt-dependent modulation of p53 could subsequently trigger a decrease of p53 promoter transactivation by p53 itself. To examine this possibility, we have transfected p53 and p53 promoter cDNAs in p19 arf-/- $p 53^{-/-}$ fibroblasts. In this cell system lacking endogenous p53, any putative activation of p53 promoter could only derive from the functional interaction between transfected p53 and p53 promoter. Indeed, Figure 3d shows that p53 overexpression activates its own promoter transactivation in $\mathrm{p} 19^{\text {arf-/- }} \mathrm{p} 53^{-/-}$ fibroblasts.

To investigate if the modulation of $\mathrm{p} 53$ by DJ-1 was also linked to additional post-transcriptional events, we have analyzed the ability of DJ-1 to affect the cellular localization of p53. As shown in Figure 2c, the overexpression of WT-DJ-1 increases p53-like immunoreactivity into the cytosol and prevents its nuclear localization, whereas both DJ-1 mutations exacerbate p53 nuclear expression. The export of p53 from nucleus to the cytosol is considered as an inactivating process because it enables p53 ubiquitination by Mdm2 and subsequent degradation by the proteasome. ${ }^{27}$ That WT-DJ-1 apparently increased cytosolic localization of p53 could be the consequence of the previously documented chaperoning 

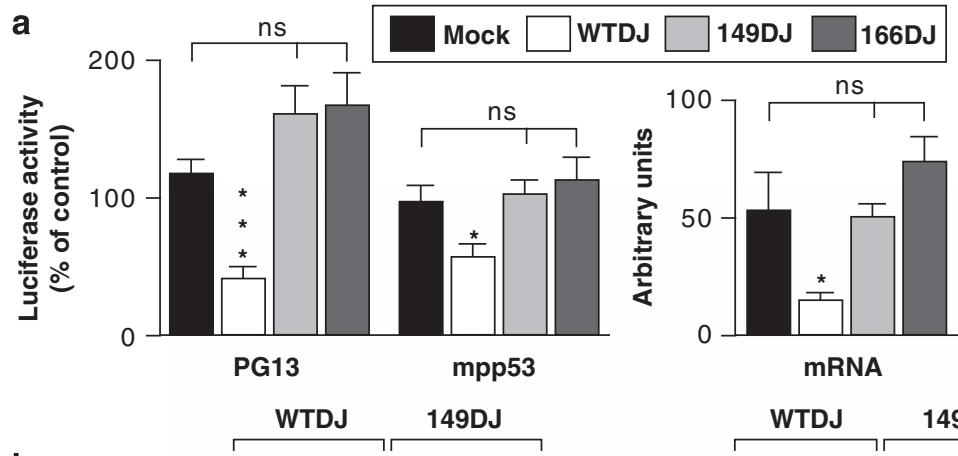

b
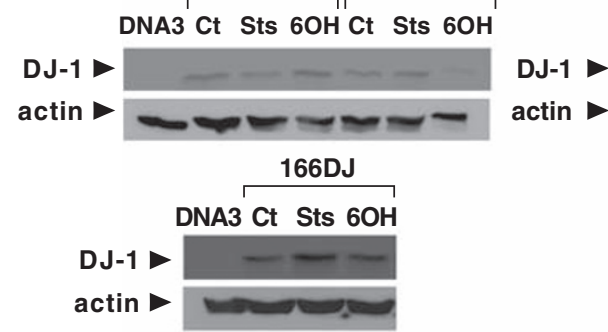

(1)

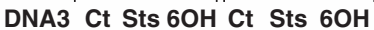

$\begin{array}{ll}\text { WTDJ } & \text { 149DJ } \\ \text { Ct Sts } 60 \mathrm{H} \text { Ct Sts } 60 \mathrm{OH}\end{array}$
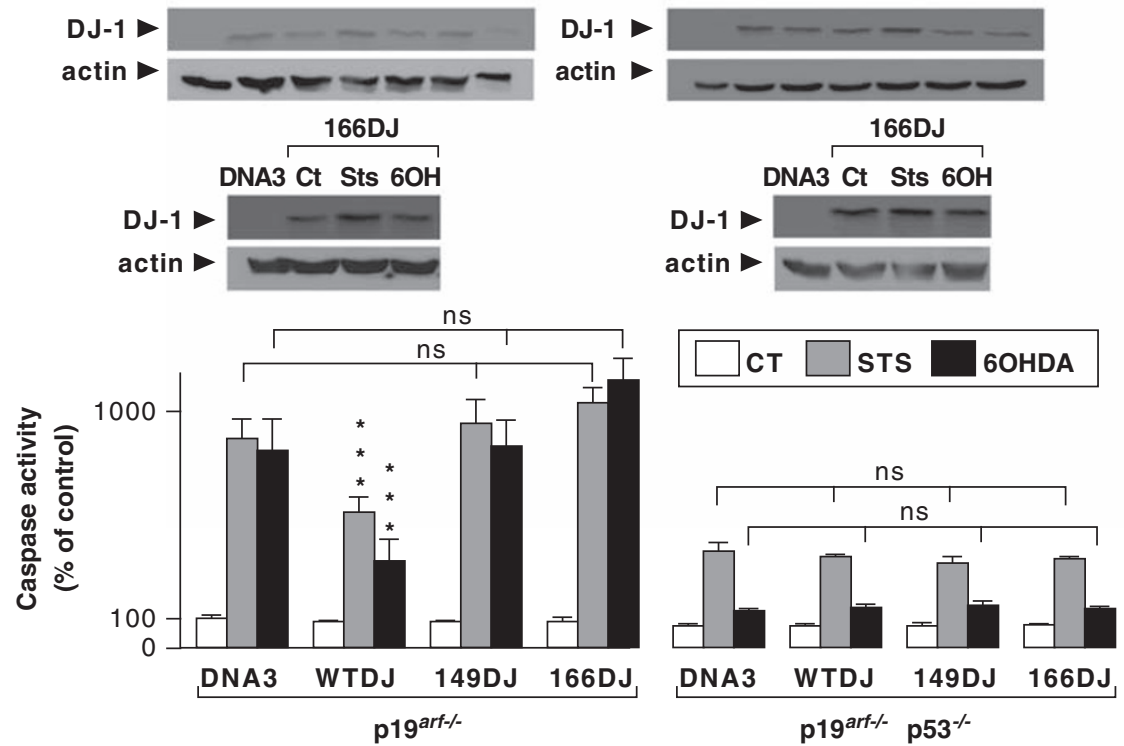

C

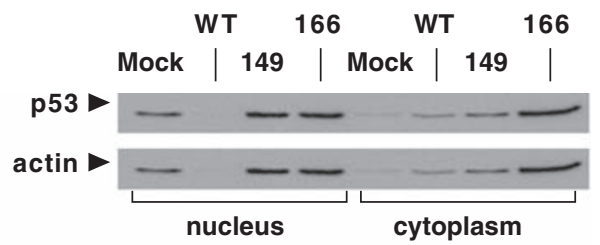

d
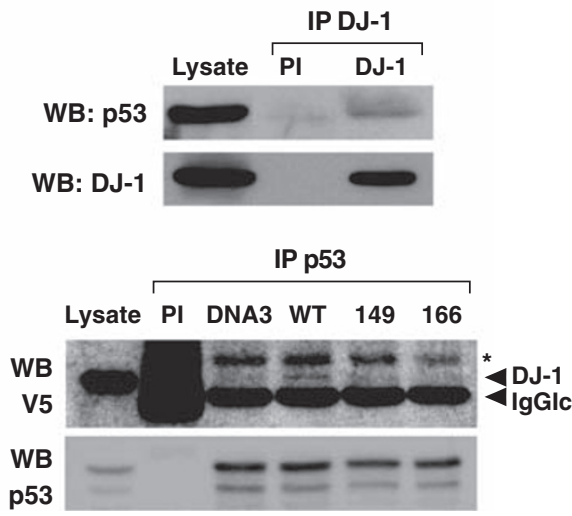

Figure 2 PD-associated mutations abolish DJ-1-associated downregulation of the p53 pathway. (a) The indicated TSM1 cell lines were monitored for their p53 activity (PG13), promoter transactivation (mpp53) and mRNA levels (mRNA) as described in the 'Materials and Methods'. Bars correspond to the means \pm S.E.M of 3-4 independent experiments performed in 3-6 replicates. ${ }^{\star} P<0.05$; ${ }^{* \star} P<0.001$. ns, not statistically significant. (b) $p 19^{\text {arf }-l-}$ and $p 19^{\text {arf }-/-} \mathrm{p} 53^{-l-}$ fibroblasts were transiently transfected with the indicated cDNA. Twenty-four hours after transfection, cells were treated for $2 \mathrm{~h}$ with STS $(2 \mu \mathrm{M})$ or $8 \mathrm{~h}$ with $6 \mathrm{OHDA}(0.2 \mathrm{mM})$ then expression of proteins $(\mathbf{b}$, upper panels) and caspase-3 activity (b, lower histograms) were monitored as described in the 'Experimental Procedure'. Bars correspond to the means \pm S.E.M of $3-5$ independent experiments. ${ }^{* \star} P<0.001$. ns, not statistically significant. (c) Nuclear and cytoplasmic expression of p53 was analyzed in the indicated stably transfected TSM1 cell lines as described in the 'Experimental Procedure'. (d) Upper panel, physical interaction between DJ-1 and p53. Endogenous DJ-1 was immunoprecipitated (IP) with anti-DJ-1 antibody or preimmune (PI) antibodies then p53- and DJ-1-like immunoreactivities were analyzed by western blot as described in the 'Experimental Procedure' (upper panel); lower panel, p53 was immunoprecipitated (IP p53) with anti-p53 antibodies then wild-type and mutated DJ-1- and p53-like expressions were analyzed by western blot as described in the 'Experimental Procedure'. *non specific protein; IgG Ic, IgG light chains

properties of $\mathrm{DJ}-1^{28}$ and could suggest a putative physical interaction between these two proteins. Coimmunoprecipitation experiments show that endogenous (Figure 2d, upper panel) and overexpressed WT-DJ-1 (Figure 2d, lower panel) physically interact with p53. Interestingly, this interaction was abolished by DJ-1 pathogenic mutations (Figure 2d, lower panel).

\section{Depletion of endogenous DJ-1 increases p53 in mouse} fibroblasts and in mice brain. To further establish the role of WT-DJ-1, we examined the contribution of endogenous DJ-1 in the control of p53-dependent cell death by means of DJ-1-deficient fibroblasts (Figure 4a, inset). Figure 4a clearly shows that the lack of DJ-1 drastically increases the STSand 6OHDA-mediated caspase-3 activation (Figure 4a, left panel, gray bars) as well as active caspase-3 immunoreactivity (Figure $4 \mathrm{a}$, right panel). It should be emphasized that DJ-1 deficiency also significantly increases caspase-3 activity in non-stimulated conditions (see CT in Figure 4a left). This observation fits perfectly with our demonstration of a significant reduction of caspase-3 activity in control conditions upon WT-DJ-1 cDNA transfection in TSM1 neurons (Figure 1b). Of most importance, we have established that this phenotype could be rescued by transient transfection of $\mathrm{DJ}-1^{-/-}$fibroblasts by WT-DJ-1 but not D149A- and L166P-DJ-1 cDNA (Figure 4b). DJ-1-deficient fibroblasts also show increased p53 transcriptional activity and mRNA levels (Figure 4c). p21 is a well known p53 gene target that is implicated in cell cycle control. ${ }^{29}$ p21 promoter sequence in frame with luciferase was therefore used as an alternative readout of p53 transcriptional activity. As expected, DJ-1 depletion increases the transcription of p21 promoter (Figure 4c). Interestingly, brain samples derived from DJ-1-deficient mice 


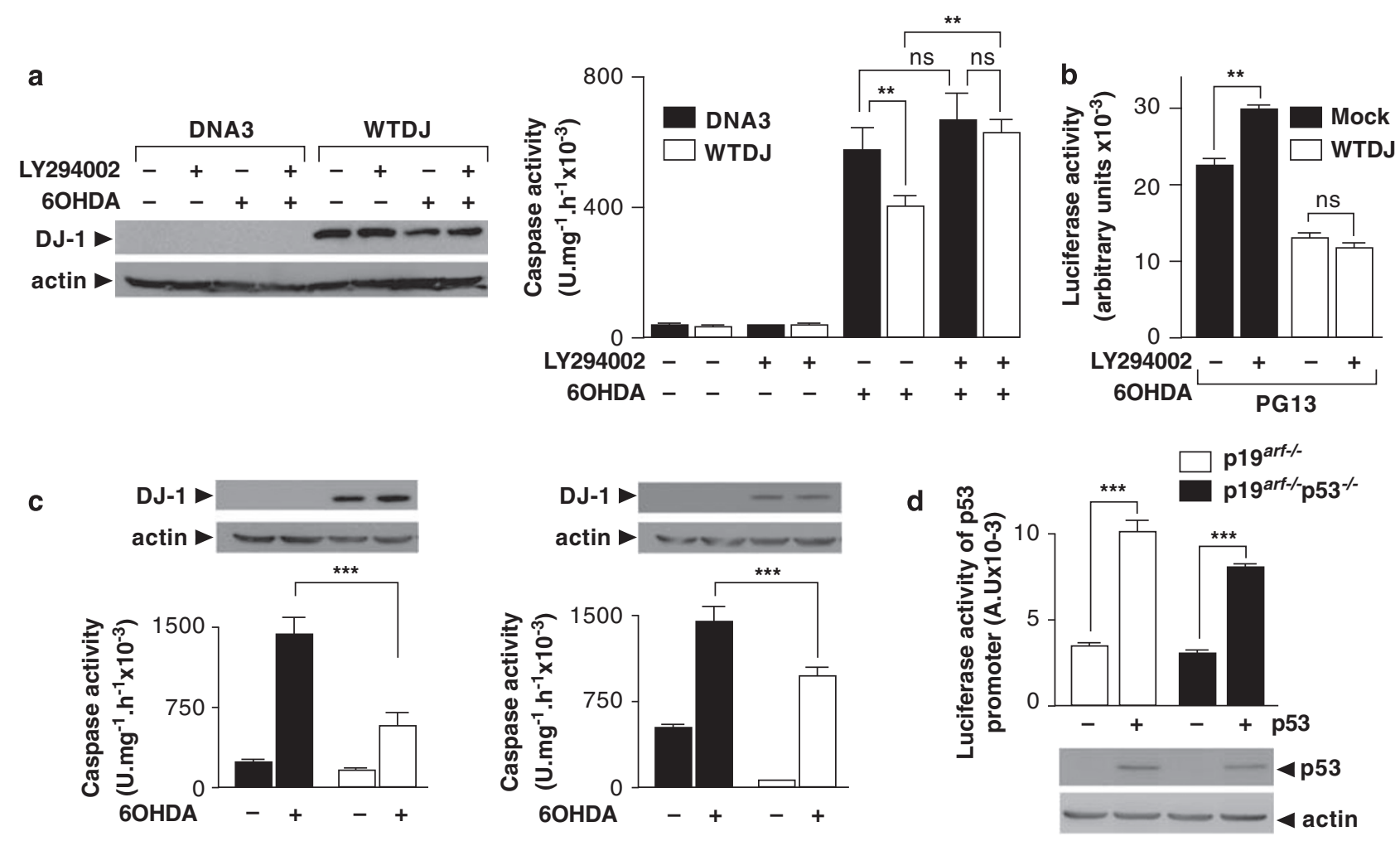

Figure 3 DJ-1 modulates p53 at a post-transcriptional level. (a) SH-SY5Y neuroblastoma cells were transiently transfected with the indicated cDNA. Twenty-four hours after transfection, cells were treated with either LY294002 $(10 \mu \mathrm{M}, 16 \mathrm{~h}), 6 \mathrm{OHDA}(0.2 \mathrm{mM}, 8 \mathrm{~h})$ or both compounds then analyzed for DJ-1 and actin expressions (left panel) and for caspase-3 activity (right panel) as described in the 'Experimental Procedure'. Bars correspond to the means \pm S.E.M of four independent experiments performed in duplicates. ${ }^{*} P<0.01$. ns, not statistically significant. (b) The indicated TSM1 cell lines were transiently transfected with the PG13 construct. Twenty-four hours after transfection, cells were treated with either LY294002 $(10 \mu \mathrm{M}, 16 \mathrm{~h})$ or vehicle then analyzed for p53 activity as described in the 'Experimental Procedure'. Bars correspond to the means \pm S.E.M of three independent experiments performed in triplicates. ${ }^{* \star} P<0.01$. (c) Wild-type (left panel) and ERK-deficient (right) fibroblasts were transiently transfected with empty pcDNA $A_{3}$ vector (black bars) or wild-type DJ-1 (empty bars) cDNAs. Twenty-four hours after transfection, cells were treated without ( - ) or with ( + ) $6 \mathrm{OHDA}(0.2 \mathrm{mM}, 8 \mathrm{~h})$ then DJ-1 expression (upper panel) and caspase-3 activity were monitored as described in the 'Experimental Procedure'. Bars correspond to the means \pm S.E.M of $6-8$ independent experiments performed in duplicates. ${ }^{* *} P<0.001$. (d) $p 19^{\text {arf }-l-}$ and $p 19^{\text {arf-l-l}} \mathrm{p} 53^{-l-}$ fibroblasts were transiently co-transfected with the mouse p53 promoter in combination with either empty pcDNA 3 or p53 cDNA. p53 promoter transactivation was monitored as described in the 'Experimental Procedure'. Bars correspond to the means \pm S.E.M of five independent experiments. ${ }^{* \star *} P<0.001$. AU, arbitrary Units

also show a mean augmentation of about $40 \%$ of p53 expression levels (Figure $4 \mathrm{~d}$ ), indicating that DJ-1 deficiency observed in vivo strictly corroborates data observed at a cellular level.

DJ-1 mutations affect DJ-1 protein stability and susceptibility to caspase- 6 proteolysis. Supplementary Figure $2 \mathrm{a}-\mathrm{c}$ clearly shows that the L166P mutation accelerates DJ-1 breakdown (note that L166P-DJ-1 is only detectable by western blot after long-term (L) exposure) whereas, conversely the D149A mutation stabilizes DJ-1 (Supplementary Figure 2c). As previously suggested, ${ }^{30}$ overexpressed WT-DJ-1 and L166P-DJ-1 catabolism is prevented by lactacystin and ALLN (Supplementary Figure $3 a, b)$ treatments, suggesting a preferential breakdown of these proteins by the proteasomal machinery. Time courses of DJ-1 expression decay, in the presence and absence of cycloheximide, confirm that the two DJ-1 mutations differently affect the stability of the neo-synthesized proteins (Supplementary Figure 2c). Therefore intriguingly, L166P and D149A DJ-1 mutations both lead to a loss of function of p53-dependent control of cell death by DJ-1 in spite of showing clearly distinct catabolic fates. If it appeared reasonable to propose that the L166P-DJ-1-associated loss of function reflects the low levels of the proteins undergoing rapid proteolysis, such exacerbated instability could clearly not account for the loss of function associated to the degradation-resistant D149A-DJ-1. Interestingly, in silico analysis of the human DJ-1 sequence revealed that this protein harbored two putative caspase- 6 consensus domains in positions $149\left(\right.$ VEKD $^{149}$ ) and $60\left(\right.$ SLED ${ }^{60}$, see Figure $\left.5 a\right)$. It is well recognized that the aspartyl (D) residue embedded in these consensus sequences is a crucial structural element strictly required for recognition/catalysis of proteins targeted by caspases. ${ }^{31}$ Therefore, the pathogenic D149A mutation described above potentially impairs a caspase- 6 cleavage site of DJ-1. In this context, we designed by site-directed mutagenesis a series of constructs either bearing single, double or triple mutations (see nomenclature in Supplementary Table 1) and we analyzed the susceptibility of the corresponding proteins to proteolysis by various recombinant caspases. Figure $5 b$ shows that WT-DJ-1, D60A-DJ-1 and L166P-DJ-1 behaved as substrates of caspase- 6 but not caspases 3 and 7. Interestingly, D149A- 

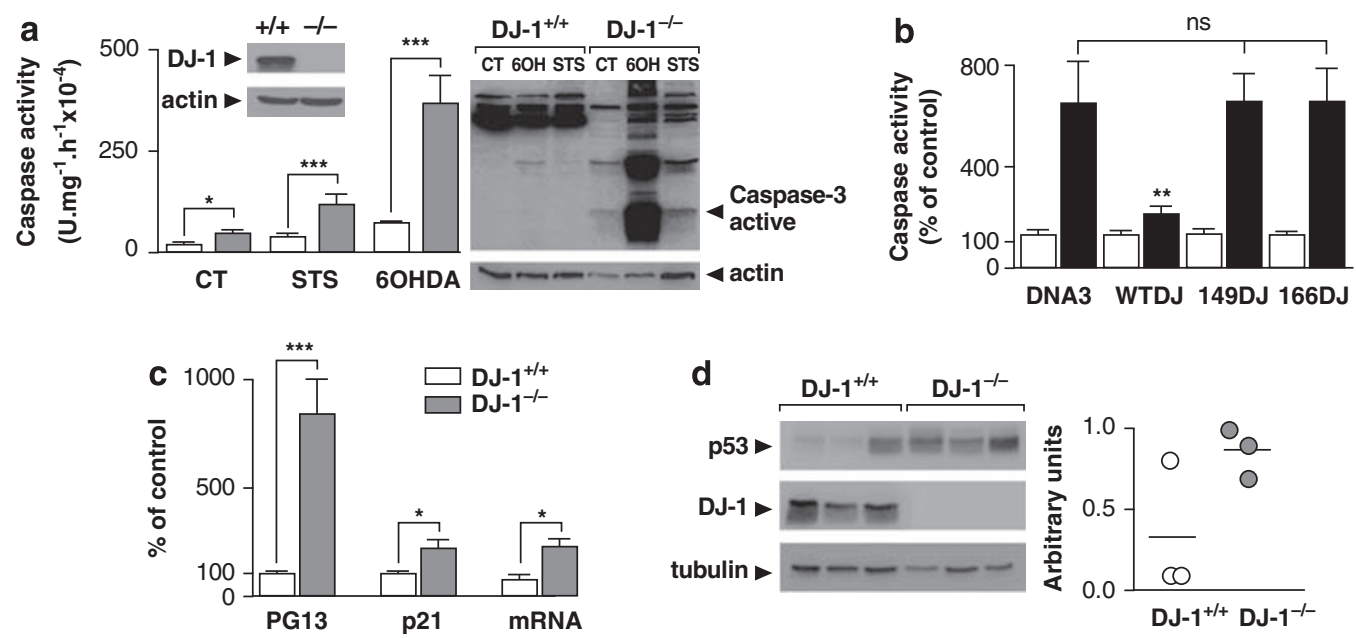

Figure 4 DJ-1 deficiency alters the p53 pathway in fibroblasts and in mice brain. (a) Wild-type (empty bars) and DJ-1 deficient fibroblasts (gray bars) were treated with STS $(2 \mu \mathrm{M}, 2 \mathrm{~h})$ or with $6 \mathrm{OHDA}(0.2 \mathrm{mM}, 8 \mathrm{~h})$ then active caspase-3 expression (right panel) and caspase-3 activity (left panel) were measured as described in the 'Experimental Procedure'. Bars correspond to the means \pm S.E.M of 3-6 independent experiments. ${ }^{*} P<0.05$; ${ }^{* *} P<0.001$. ns, not statistically significant. (b) DJ-1-deficient fibroblasts were transiently transfected with the indicated cDNA as described in the 'Experimental Procedure'. Twenty-four hours after transfection, cells were treated for $8 \mathrm{~h}$ with vehicle (white bars) or with $6 \mathrm{OHDA}(0.2 \mathrm{mM}$, black bars) then caspase-3 activity was monitored as above. Bars correspond to the mean of \pm S.E.M of 12-16 independent experiments. ${ }^{*} P<0.01$. ns, not statistically significant. (c) Wild-type and DJ-1-deficient fibroblasts were monitored for their p53 activity (PG13) and mRNA levels (mRNA) as well as for the transactivation of the promoter of p21 as described in the 'Experimental Procedure'. Values are expressed as the percent of control activities obtained in wild-type fibroblasts (taken as 100) and are the means \pm S.E.M of 3-4 independent experiments performed in six ( PG13 and p21) or three (mRNA levels) replicates. ${ }^{*} P<0.05 ;{ }^{* \star *} P<0.001$. (d) Expression patterns (left) and densitometric analyses (right) of endogenous DJ-1 and p53 in wild-type (DJ-1 ${ }^{+/+}$) and DJ-1 knockout (DJ- $1^{-/-}$) mice brain extracts were measured as described in the 'Experimental Procedure'

a

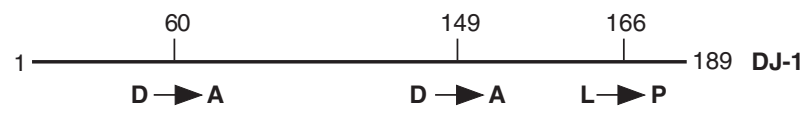

b

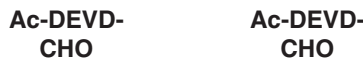

C

Ac-DEVDCHO $\mathrm{CHO}$

СТ С $\mathrm{C} 6 \mathrm{C} 7 \mathrm{C} 3 \mathrm{C} 6 \mathrm{C} 7 \mathrm{CT}$ C3 $\mathrm{C} 6 \mathrm{C} 7 \mathrm{C} 3 \mathrm{C} 6 \mathrm{C} 7$
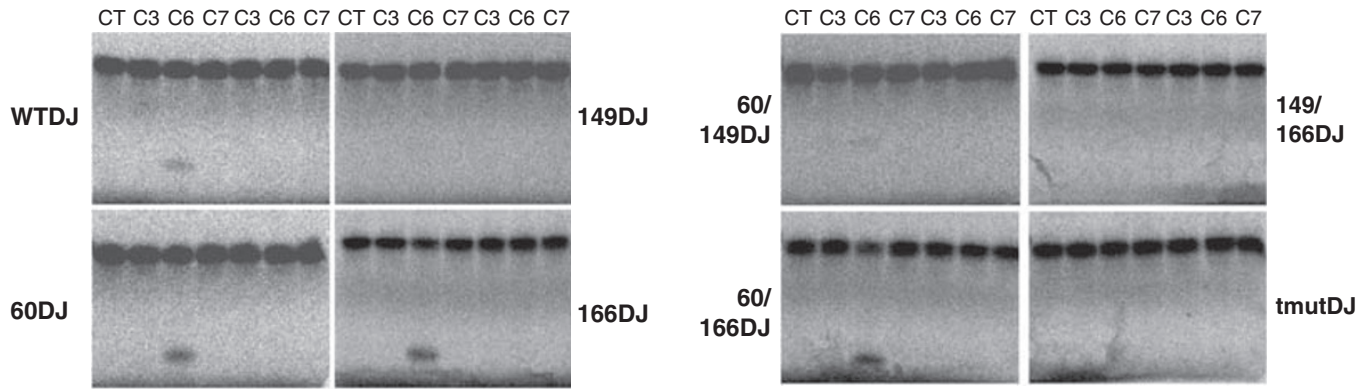

Figure 5 Distinct susceptibility of wild-type and mutated DJ-1 to proteolysis by caspases. (a) Localization and nature of the three different pathogenic point mutations on DJ-1. DJ-1 mutants harboring one (60DJ, 149DJ or 166DJ), two (60/149DJ, 60/166DJ or 149/166DJ) or three (tmutDJ) mutations (for nomenclature see Supplementary materials Table 1) were obtained by site-directed mutagenesis as described in the 'Experimental procedure' (A, alanine; $D$, aspartic acid; L, leucine; P, proline). (b-c) The indicated constructs were transcribed and translated in vitro with ${ }^{51}$ methionine and incubated for $8 \mathrm{~h}$ at $37^{\circ} \mathrm{C}$ with purified recombinant caspase-3, -6 and -7 (C3, 6,$7 ; 25 \mathrm{ng}$ ) in the absence or presence of the caspase inhibitor (Ac-DEVD-CHO,10 $\mu \mathrm{M}$ )

DJ-1 fully resisted proteolysis by caspase-6 (Figure 5b) whereas the L166P mutation apparently enhanced DJ-1 susceptibility to recombinant caspase-6 (Supplementary Figure 2b). Analysis of double and triple mutations (tmut) confirmed that the alanine substitution at position 149 fully blocked the cleavages of 60/149DJ, 149/166DJ and tmutDJ by caspase-6, whereas 60/166 remained cleaved by recombinant caspase-6 (Figure $5 \mathrm{c}$ ). These data show that $\mathrm{DJ}-1$ is cleaved by caspase- 6 at only one out of the two putative caspase-6 consensus sequences located at positions 146-149.

The caspase-6-derived C-terminal product of DJ-1 accounts for DJ-1-associated protective phenotype. A question remained as to whether the loss of function triggered by the D149A mutation could be fully due to the impairment of DJ-1 proteolysis by caspase-6. If true, one would predict that one of the caspase-6-derived proteolytic 
ns
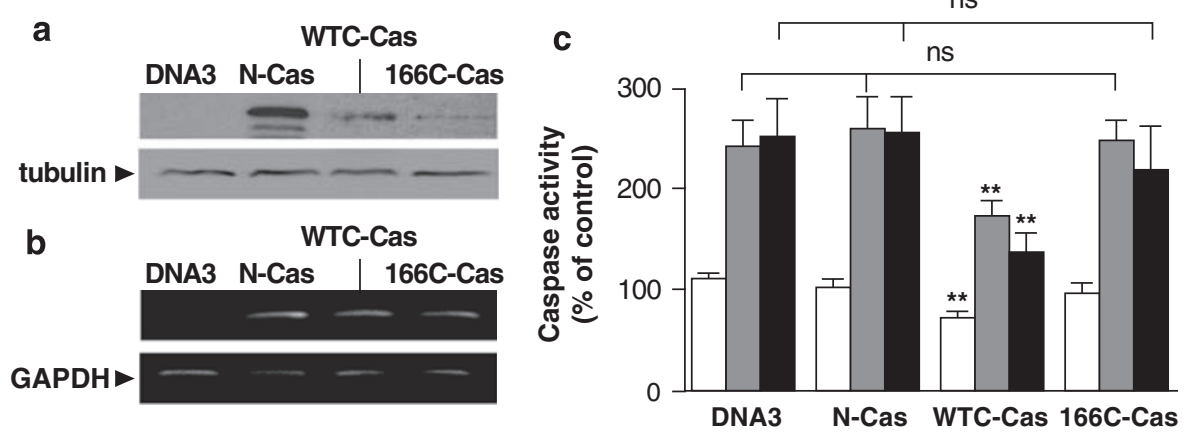

d WTC-Cas
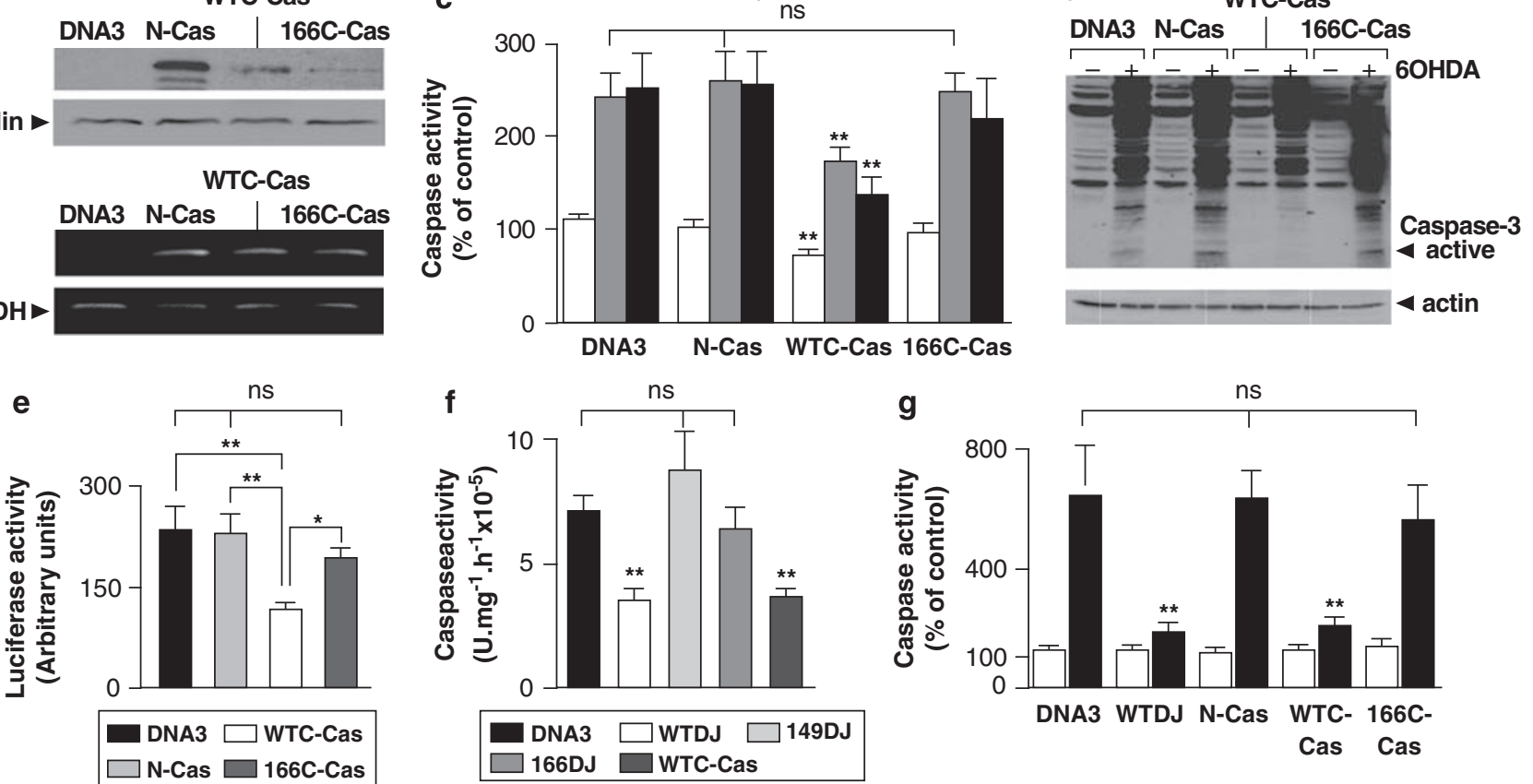

Figure 6 The caspase-6-derived C-terminal fragment of DJ-1 lowers caspase-3 activation and p53 activity. (a-b) HEK293 cells were transiently transfected with the indicated cDNA (see Supplementary material Table 1). Expression (a) and mRNA levels (b) of DJ-1 fragments were analyzed by western blot with anti-V5 antibodies (a) or by RT-PCR with V5-specific primers (b) as described in the 'Experimental procedure'. (c-d) HEK293 cells were transiently transfected with the indicated cDNA. Twenty-four hours after transfection, cells were treated with STS $(2 \mu \mathrm{M}, 16 \mathrm{~h}$, gray bars) or with 6OHDA (0.2 mM, $8 \mathrm{~h}$, black bars) then caspase- 3 activity (c) and active caspase-3 immunoreactivity (d) were monitored as described in the 'Experimental Procedure'. Bars in c represent the means \pm S.E.M of 4-5 independent experiments performed in duplicates and are expressed as percent of control caspase-3 activity obtained in untreated mock-transfected cells. ${ }^{* *} P<0.01$ (comparison between WTC-Cas versus Mock, $\mathrm{N}$-Cas and 166C-Cas). ns, not statistically significant. (e) HEK293 cells were co-transfected with PG13-luciferase cDNA and the indicated cDNA then p53 activity was measured as described in the 'Experimental Procedures'. Bars correspond to the means \pm S.E.M of four independent experiments performed in six replicates. ${ }^{\star} P<0.05$; ${ }^{\star *} P<0.01$. ns, not statistically significant. (f) Primary neurons were transiently transfected by nucleoporation with the indicated cDNAs as described in 'Experimental Procedures'. Four days after transfection, neurons were treated for $16 \mathrm{~h}$ with STS $(2 \mu \mathrm{M})$ then caspase-3 activity was monitored as described in 'Experimental Procedures'. Bars are the means \pm SEM of five independent experiments corresponding to the STS-stimulated caspase-3 activity. ${ }^{\star \star} P<0.01$. ns, not statistically significant. (g) DJ-1deficient cells were transiently transfected by nucleoporation with the indicated cDNA as described in 'Experimental Procedures'. Twenty-four hours after transfection, cells were treated for $8 \mathrm{~h}$ with vehicle (white bars) or with 6 OHDA $(0.2 \mathrm{mM}$, black bars) then caspase- 3 activity was monitored as above. Bars correspond to the means \pm S.E.M of 10-16 independent experiments and are expressed as percent of control caspase-3 activity obtained in mock-transfected fibroblasts. ${ }^{\star \star} P<0.01$. ns, not statistically significant

fragments of DJ-1 should bear its protective function. We have designed, by site-directed mutagenesis, the $\mathrm{N}$-terminal (N-Cas) and C-terminal (WTC-Cas) fragments of WT-DJ-1 theoretically derived from its cleavage after residue 149. Furthermore, we have obtained the caspase-6-derived fragment of L166P-DJ-1 referred to as 166C-Cas (see Supplementary Table 1). Figure $6 a$ shows the expression profile of the proteins derived from these constructs after transient transfection in human embryonic kidney 293 cells (HEK293) cells. In agreement with previous considerations, we observed a rather poor expression of 166C-Cas (Figure 6a). This prompted us to analyze 166C-Cas mRNA levels to rule out the possibility that distinct transfection efficiencies could interfere with interpretation of the data. Figure $6 b$ shows that mRNA levels were similar in all transfection experiments. Therefore, the L166P mutation indeed drastically enhances the catabolic instability of both entire and C-terminal fragments. It should be emphasized that the WTC-Cas fragment was also recovered at lower levels than the N-terminal counterpart (Figure 6a), suggesting that the stability of DJ-1 was clearly conditioned by its C-terminal moiety. This agrees well with the fact that DJ-1 homodimerization was reported to occur through the association of the $\mathrm{C}$-terminal part of the proteins and that the L166P impairs such association. ${ }^{15}$

Figure 6c shows that transient transfection of HEK293 cells with the WTC-Cas cDNA triggers a drastic reduction of caspase-3 activity in non-stimulated conditions (empty bars) as well as in STS- (gray bars) or 6OHDA- (black bars) stimulated conditions whereas $\mathrm{N}$-Cas remained totally inactive. Interestingly, the WTC-Cas-mediated response was abolished by the L166P mutation (Figure 6c). This phenotype was further confirmed by the analysis of the levels of active caspase-3 in the same transfected samples (Figure 6d). Thus, WTC-Cas also triggers a significant reduction of the number of tunel-positive apoptotic nuclei (Supplementary Figure 4). Of most interest, WTC-Cas was also the only fragment of DJ-1 able to lower p53 transcriptional activity (Figure 6e). Of importance, the protective effect associated to DJ-1 and WTC-cas was also observed in primary cultured neurons (Figure 6f). We confirmed the protective function harbored by the WTC-Cas by complementation experiments in DJ-1- 
deficient fibroblasts. Figure $6 \mathrm{~g}$ illustrates the influence of WTDJ, N-Cas, WTC-Cas and 166C-Cas after transient transfection of DJ-1 ${ }^{-1-}$ fibroblasts. We show that only WTDJ and its caspase-6-derived fragment WTC-Cas similarly reduced caspase-3 activation whereas $\mathrm{N}$-Cas and $166 \mathrm{C}$ Cas remained biologically inactive. Overall, our data show that the caspase-6-derived C-terminal product of DJ-1 fully accounts for the protective phenotype elicited by DJ-1. We have examined whether 6OHDA could increase caspase-6 activity and, subsequent to this activation, whether an enhanced production of endogenous WTC-Cas could be evidenced. Supplementary Figure 5 shows that there exists both constitutive and 6OHDA-stimulated caspase- 6 activity that can be prevented by the selective caspase- 6 inhibitor $N$ Acetyl-Val-Glu-Ile-Asp-al (Ac-VEID-CHO) (Supplementary Figure 5a). Interestingly, we were able to precipitate WCTCas after 6OHDA stimulation in SH-SY5Y cells, the production of which was fully prevented by Ac-VEID-CHO (Supplementary Figure $5 \mathrm{~b}$ ). Furthermore, by mass spectroscopy analysis (Supplementary Figure 5) we showed that 6OHDA increases the recovery of a product corresponding to the expected WTC-Cas mass (Supplementary Figure 5c, lower panel, inset) that was absent in vehicle-treated samples (Supplementary Figure 5c, upper panel, inset). It should be noted that both approaches show that endogenous levels of WTC-Cas are relatively weak, agreeing well with our demonstration of its catabolic instability (see Figure 6a). Altogether, these data suggest that in experimental conditions mimicking PD pathology, caspase- 6 could be activated, thereby increasing the cleavage of endogenous DJ-1 and subsequent production of WTC-Cas product.

Altered processing of $\mathrm{DJ}-1$ in sporadic PD brains. To examine whether our cellular data could be extrapolated to a

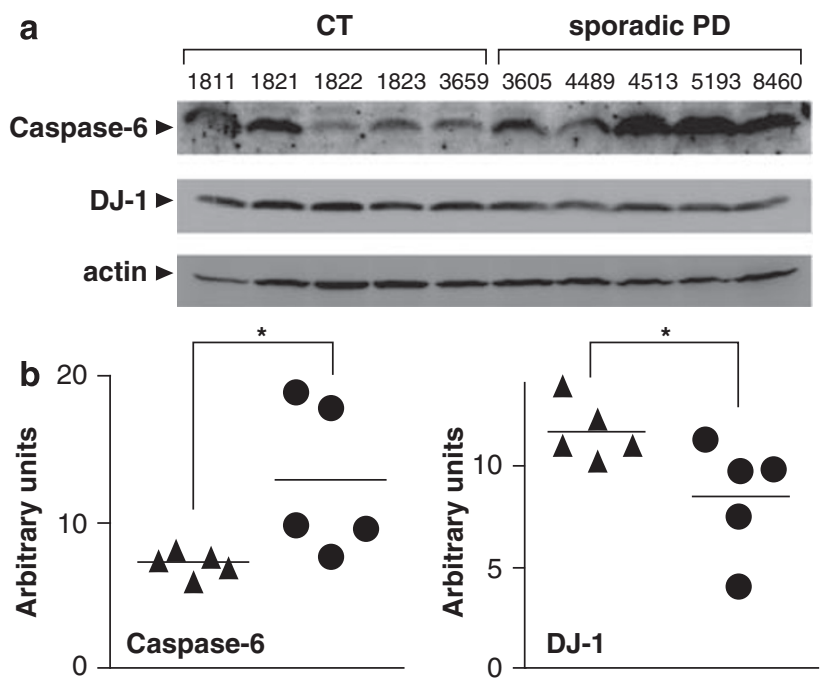

Figure 7 Caspase- 6 and DJ-1 levels are altered in the substantia nigra of sporadic Parkinson's disease -affected human brains. Caspase-6 and DJ-1 expressions (a) and densitometric analyses (b) in control (CT, triangles, see Experimental Procedure) and sporadic Parkinson's disease (PD, dots) human brains were analyzed as described in the 'Experimental Procedures'. Each point in $B$ corresponds to three independent determinations. ${ }^{*} P<0.05$ pathological context, we analyzed the levels of DJ-1 and caspase- 6 in human brains obtained from control and sporadic PD patients. As depicted in Figure 7a,b, the levels of caspase-6 and DJ-1 appeared inversely correlated in normal and PD-affected brains, suggesting that increased expression of caspase- 6 activity could explain the slight but statistically significant reduction of DJ-1 levels observed in pathological brains.

\section{Discussion}

Several studies have consistently documented a protective role of DJ-1 against various pro-apoptotic effectors or stressinducing agents ${ }^{32}$ but the mechanisms underlying such function still remained barely known. Here, we show that the overexpression of DJ-1 lowers STS- and 6OHDA-induced caspase-3 activation via a reduction of p53 expression and activity in various cell systems including primary cultured neurons, TSM1 neurons, SH-SY5Y neuroblastoma, HEK293 cells and mouse fibroblasts. Of most importance, the depletion of endogenous DJ-1 triggers an opposite phenotype that can be rescued by complementation of these cells with the wild-type DJ-1 construct. To our knowledge, this is the first demonstration that $\mathrm{DJ}-1$ reduces the p53 pathway in human and neuronal cells in response to various stimuli, including 6OHDA, a naturally occurring toxic dopaminergic by-product. ${ }^{33}$ These data agree well with another work showing that DJ-1 depletion triggers increased Bax and p53 expressions in the zebrafish. ${ }^{34}$

Two distinct lines of data indicate that the DJ-1-associated phenotype is fully dependent of p53 and involves posttranscriptional control of this tumor suppressor. First, DJ-1-associated protective function is fully abolished by p53 deficiency. Second, DJ-1 controls p53 by activating Aktdependent and Erk and $\mathrm{NF}_{\kappa} \mathrm{B}$-independent pathways and by triggering its sequestration within the cytosol. These data agree well with a previous study documenting the fact that downregulation of DJ-1 levels by siRNA targeting approaches lead to reduced Akt phosphorylation, in vivo. ${ }^{35}$ Interestingly, DJ-1 also lowers p53 promoter transactivation. This appeared paradoxical with respect to our data showing that the pharmacological blockade of the Akt pathway fully prevents DJ-1-associated phenotype. However, in agreement with previous data, ${ }^{36}$ our demonstration that p53 could activate its own transcription in fibroblasts (Figure $3 \mathrm{c}$ ) suggests that this phenomenon could likely account for DJ-1-induced decrease of p53 promoter transactivation.

Although most of the cases of PD are of sporadic origin, it remains that the delineation of the molecular dysfunctions responsible for a rather low subset of genetic cases should help in understanding PD pathogenesis. To date, only one deletion and one point mutation in the gene of $\mathrm{DJ}-1$ have been reported in two distinct families. In the latter case, the replacement of a leucine residue in position 166 by a proline introduces a strong helix breaking element that precludes DJ1 homodimerization, ${ }^{15}$ increases catabolic instability and thereby, impairs its biological activity. More recently, another mutation that replaces an aspartyl residue in position 149 by an alanine was reported to trigger early-onset PD although the 
family history is not yet available to ascertain that transmission of this mutation results from a recessive transmission.

At a macroscopic level, the two DJ-1 mutations led to similar impairment of DJ-1-mediated antiapoptotic phenotype. Thus, both L166P and D149A mutations abolish DJ-1-induced reduction of STS- and 6OHDA-stimulated caspase-3 activity and were unable to rescue DJ-1 phenotype in complementation experiments performed in DJ-1-deficient fibroblasts. On the other hand, it clearly appeared that unlike D149A-DJ-1 that behaves as the parent protein, L166P-DJ-1 displayed a remarkable short life in our cells. The above observations suggested that mutations at residues 149 and 166 similarly impair DJ-1 protective function through distinct mechanisms.

The fact that an aspartyl residue substitution was apparently responsible for DJ-1 dysfunction led us to envision that the D149A mutation could have prevented DJ-1 catabolism by either aspartyl proteases or cystein caspase-like activities. Indeed, in silico analysis of DJ-1 sequence identified two consensus sites for caspase- 6 activity corresponding to SLED and VEKD tetrapeptides where aspartyl residues were in positions 60 and 149, respectively. Thus, we envisioned the possibility that the D149A mutation could impair DJ-1 proteolysis and, as a corollary, that such resistance to caspase-6-mediated cleavage could be responsible for the loss of function triggered by the mutation. Five distinct lines of evidence support this hypothesis. First, DJ-1 was readily proteolysed by recombinant caspase- 6 but not caspases-3 and -7 . Second, the D149A but not the D60A mutation fully impaired DJ-1 cleavage by caspase- 6 . Third, the C-terminal fragment of DJ-1 (WTC-Cas) that would theoretically derive from its processing by caspase- 6 at site 149 mimics DJ-1 phenotype, reduces STS- and 6OHDA-stimulated caspase-3 activity and lowers p53 transcriptional activity. Fourth, WTCCas fully rescues DJ-1 phenotype after complementation experiments in DJ-1-deficient fibroblasts. Fifth, endogenous WTC-Cas is produced upon 6OHDA challenge and this generation is prevented by the caspase- 6 inhibitor Ac-VEID$\mathrm{CHO}$. Altogether, these data show that the C-terminal moiety of DJ-1 corresponding to WTC-Cas is responsible for DJ-1associated protective phenotype and that this function is abolished by the D149A mutation that impairs caspase-6mediated cleavage of DJ-1. Thus, our study is the first demonstration that $\mathrm{DJ}-1$ antiapoptotic function is controlled by its proteolysis by caspase-6. It is interesting to note that synphilin-1, a physiological partner of $\alpha$-synuclein ${ }^{37}$ also undergoes caspase-mediated biological maturation. Thus, we established recently that synphilin-1 is readily targeted by caspase- 3 and poorly processed by caspases- 6 and $-7 .^{38}$ Interestingly, the C-terminal fragment of synphilin-1 generated by caspase- 3 harbored a protective function by controlling the p53-dependent pathway. ${ }^{38}$

Overall, these data and our present work reinforced the current view that the p53-dependent cell death could be a central process in PD pathology ${ }^{39,40}$ but also unmask the crucial involvement of caspase-dependent proteolytic events in the function of PD-associated proteins and suggest that distinct caspases could be specifically involved in the physiological maturation of these proteins. On the other hand, caspase-mediated cleavages could be seen as inactivating mechanisms. Thus, parkin undergoes proteolysis by cas- pases 1 and $8^{41}$ leading to an impairment of its ubiquitin-like domain (UBL). This process inactivates the parkin-associated E3-ligase activity that controls the levels of ubiquitinated CDCrel-1 and Pael- $\mathrm{R}^{42,43}$ and therefore its subsequent proteasomal degradation. Overall, the above studies along with our present work reveal the crucial involvement of caspase-dependent proteolytic events in the function of PDassociated proteins and suggest that distinct caspases could be specifically involved in the physiological maturation and/or inactivation of these proteins.

Our study is also the very first report showing that various pathogenic mutations could trigger DJ-1 loss of function via totally distinct molecular mechanisms, that is, protein destabilization or resistance to caspase-6-mediated proteolysis (see Figure 8). In line with our data, a recent study also indicates that parkin mutations impair its E3-ligase activity by triggering either misfolding or destabilization of the protein. ${ }^{44}$ The loss of the E3-ligase activity likely results in an impairment of parkin function because the deletion of the UBL domain of

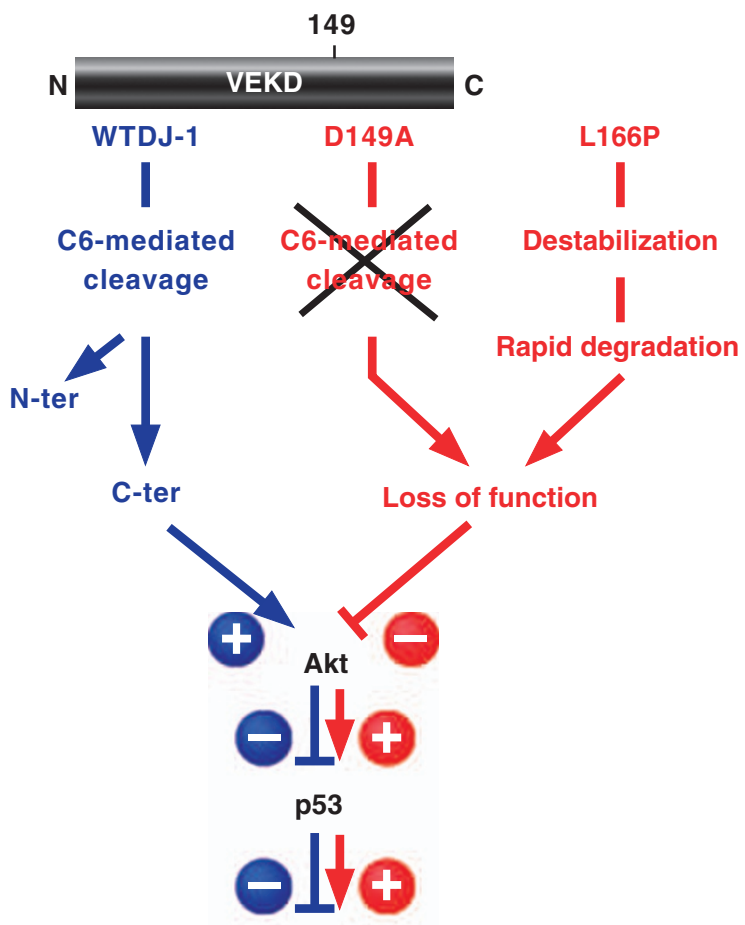

Caspase-3 activation

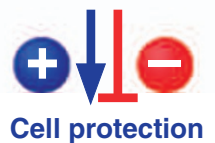

Figure 8 Model of DJ-1-associated cell protection and its alteration by pathogenic mutations. Caspase- 6 activation by a cellular stress triggered by staurosporine or the natural dopaminergic toxin 6OHDA increases caspase-6mediated cleavage of wild-type DJ-1, thereby generating a C-terminal DJ-1 fragment that activates Akt, reduces p53 activity and therefore acts as a protective cellular signal (blue pathway). Mutation of DJ-1 at amino-acid 149 prevents its cleavage by caspase-6. A DJ-1 mutation at position 166 destabilizes DJ-1 and renders the protein prone to rapid degradation. Both mutations trigger a loss of function of DJ-1 and abolish DJ-1-mediated control of p53 (red pathway). Therefore, various mutations of DJ-1 can be likely responsible for Parkinson's disease pathology via distinct molecular dysfunctions 
parkin compromises its ability to protect cells against ceramide-induced toxicity. ${ }^{45}$

Of most interest, we have established that the levels of endogenous caspase- 6 in normal and sporadic PD were inversely correlated (see Figure 7). This strongly corroborates our view that caspase- 6 is indeed a physiological modulator of endogenous DJ-1 levels in human brain and agrees well with our data showing that the dopaminergic toxin 6OHDA increases cellular caspase- 6 activity and enhances the cleavage of endogenous DJ-1 and production of its C-terminal catabolite WTC-Cas. Therefore, one could envision that the increased caspase- 6 expression observed in sporadic PD could be seen as a putative compensatory mechanism aimed at interfering with the PD-associated degenerative process. According to our data and together with the above-cited previous reports, it is tempting to suggest that pathogenic mutations differently affecting caspase-6-mediated proteolysis of DJ-1 could lead to distinct disease onsets and phenotypes as it was observed for Parkinson's disease patients harboring distinct parkin mutations. ${ }^{44}$

\section{Materials and Methods}

Antibodies and materials. The anti-V5 mouse monoclonal antibody and lipofectamine reagent were purchased from Invitrogen (Invitrogen Corporation, CA, USA). Anti-DJ-1 rabbit polyclonal antibodies (ab18257 and ab37180 used for western blot and coimmunoprecipitation analysis, respectively) and anti-caspase- 6 monoclonal antibodies (ab17866) were purchased from Abcam (Abcam, Paris, France). Anti-human total p53 antibodies were purchased from Santa Cruz Biotechnology (Santa Cruz, CA, USA). Anti- $\beta$-tubulin and anti-actin monoclonal antibodies, STS, 6OHDA, cycloheximide, Ac-DEVD-aldehyde, Ac-VEID-CHO, Ac-DEVD-7-amino-4-methylcoumarin, ALLN ( $N$-acetyl-L-leucyl-L-norleucinal), AEBSF [4-(2-aminoethyl) benzenesulphonyl fluoride hydrochloride], E-64 [trans-epoxysuccinyl--L-leucylamido-(4-guanidino)butane], lactacystin, pepstatin, o-phenanthroline and calpain inhibitor I were purchased from Sigma (St QuentinFallavier, France). LY294002 was purchased from Cayman Chemicals (Ann Arbor, MI, USA). The IkB kinase inhibitor BMS-345541 was purchased from Calbiochem (Merck Chemicals Limited, Nottingham, England). Fluorogenic substrate for caspase-6 the 7-amino-4-trifluoromethyl coumarin was purchased from Biomol (BIOMOL International, Plymouth, PA, USA). Anti-human PARP antibodies were purchased from Euromedex (Souffelweyersheim, France). The anti-active caspase3 rabbit polyclonal antibody was purchased, R\&D Systems (R\&D Systems, Minneapolis, USA). S17092 (Barelli et al., 1999) was kindly provided by Philippe Morain (Servier, Paris). Benzylocarbonyl-lle-Leu-(OBut)-Ala-leucinal and benzyloxycarbonyl-leucinal were kindly provided by Dr S Wilk (Mount Sinaï, New York, USA)

Site-directed mutagenesis. D149A-DJ-1 and D149A/L166P-DJ-1 were obtained by site-directed mutagenesis from wild-type DJ-1 and mutated L166PDJ-1 V5-tagged CDNA by means of QuikChange site-directed mutagenesis kit (Stratagene, La Jolla, CA, USA). The two primers containing the D149A pathogenic mutation $5^{\prime}$-CGTGTGGAAAAAGCAGGCCTGATTCTTACAAGC- $3^{\prime}$ and 5'-GCTTGTAAGAATCAGGCCTGCTTTTTCCACACG-3' (Eurogentec, Angers, France) were designed according to the manufacturer's recommendations. The D60A-DJ-1, the D60A/D149A-DJ-1 and the D60A/D149A/L166P-DJ-1 were obtained by oligonucleotide-directed mutagenesis from wild-type DJ-1, mutated D149A-DJ-1, and mutated D149A/L166P-DJ-1 V5-tagged cDNAs by means of QuikChange site-directed mutagenesis kit (Stratagene). The two primers containing the D60A mutation were as follows: $5^{\prime}$-GCCTTGAAGCAGCAAAAAAAGAGGG-3' and $5^{\prime}$-CCCTCTTTTTTTGCTGCTTCAA GGC-3' (Eurogentec) were designed according to the manufacturer's conditions. The CDNA encoding the V5-tagged caspase-6-derived $\mathrm{N}$-terminal fragment of DJ-1 was engineered by introducing a Xho restriction site in position 150, that is, after the aspartyl residue in position 149 involved in a putative consensus cleavage site of caspase-6 (oligonucleotide $5^{\prime}$-CTAGACTCGAGTCTTTCACACG-3'), to allow the subcloning of the construction in pcDNA3.1/V5/His-TOPO. The C-terminal fragment of DJ-1 was obtained by introducing an ATG codon in position 149 (oligonucleotide 5'-TAATTAAGCTTATG GGCCTGATTCTTACAAGCCGG-3'). An additional Hindlll restriction site was also added, adjacent to the ATG codon, for further subcloning of the construction in pcDNA3.1/V5/His-TOPO.

Cell systems and transfections. Telencephalon-specific mouse 1 (TSM1), HEK293, SH-SY5Y human neuroblastoma cells and mouse embryonic fibroblasts (MEF) were cultured in Dulbecco's media supplemented with $10 \%$ fetal bovine serum. Stable transfectants expressing empty vector, wild-type and mutated DJ-1 in TSM1 cells were obtained after transfection with $2 \mu \mathrm{g}$ of each cDNA (all in pcDNA3) by means of Lipofectamine according to the manufacturer's conditions. Positive clones were screened for their DJ-1-like immunoreactivity and mRNA levels as described below. Transient transfections of SH-SY5Y, DJ-1 knockout MEF cells, ${ }^{6}$ p1 $19^{\text {Arf }}$-deficient and p19 $19^{\text {Arf }} / \mathrm{p} 53$ double knockout MEF cells ${ }^{46}$ and ERK-1 knockout MEF cells ${ }^{47}$ were carried by means of either lipofectamine reagent or Nucleofector kit according to the manufacturer's instructions (Amaxa Biosystems, Koeln, Germany) as previously described. ${ }^{48}$

Tunel analysis of cell death. SH-SY5Y human neuroblastoma cells were transfected with empty pcDNA3 vector or different DJ-1 cDNAs by means of the Lipofectamine reagent according to the manufacturer's conditions and cultured in polylysinated cover-slips. Twenty-four hours after transfection, cells were treated with or without $60 \mathrm{HDA}(0.2 \mathrm{mM}, 8 \mathrm{~h})$ then fixed for $30 \mathrm{~min}$ with $4 \%$ paraformaldehyde, rinsed in PBS, permeabilized overnight with $70 \%$ ethanol and then processed for the dUTP nick-end labeling (Tunel) technique as described previously. ${ }^{49}$ Staining was assessed with peroxidase-conjugated antibody and revealed with a diaminobenzidine substrate as described. ${ }^{49}$ Fragmented DNA labeling corresponds to black spots. The visualization of the totality of the cells was carried out with erythrosin B.

Primary cultures of neurons. Embryonic cortical neurons were prepared as previously described (Vincent et al., 1996). Briefly, cells from cerebral hemispheres of C57B mouse embryos (E14 stage) were dissociated in Ham's F12 (Fisher Bioblock Scientific, Illkirch, France), supplemented with $0.6 \%$ glucose and $10 \%$ fetal calf serum. A total of $5 \times 10^{6}$ cells were transfected with $3 \mu \mathrm{g}$ of each cDNA (all in pcDNA3) by using a Mouse Neuron Nucleofector kit according to the manufacturer's instructions (Amaxa Biosystems, Koeln, Germany) and were seeded in 12-well plates pre-coated with $10 \mu \mathrm{g} / \mathrm{ml}$ polylysine (Sigma).

Western blot analyses. Cells were homogenized in lysis buffer $10 \mathrm{mM}$ Tris-

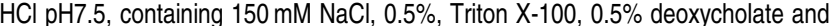
$5 \mathrm{mM}$ EDTA and resolved on 12\% SDS-PAGE for the analysis of wild-type and mutated tagged-DJ-1, endogenous DJ-1, total p53 and active caspase- 3 or on $8 \%$ SDS-PAGE for the detection of PARP. For analysis of the immunoreactivities of the $\mathrm{N}$ - and $\mathrm{C}$-terminal fragments of DJ-1, proteins were separated on $16.5 \%$ Tris/tricine gels. After electrophoresis, proteins were wet transferred to nitrocellulose membranes and incubated overnight with the adequate primary antibodies (see above). Protein immunoreactivities were revealed with either an anti-rabbit peroxidase or an anti-mouse peroxidase (Jackson Immunoresearch, Cambridgeshire, UK) by the electrochemiluminescence method as previously reported. ${ }^{50}$ Chemiluminescence was recorded using a luminescence image analyzer LAS-3000 (Raytest, Courbevoie, France), and quantification of captured images was performed using the Aida Image Analyzer software (Raytest).

In Vitro transcription/translation of wild-type and mutated DJ-1 and cleavage by recombinant caspase- $3,-6$ and -7 in a cell-free system. Wild-type and mutated DJ-1 were transcribed and translated by means of the Promega (Charbonnieres, France) TNT-coupled reticulocyte lysate system ${ }^{51}$ in the presence of radiolabeled methionine (ICN) as described. ${ }^{38}$ Briefly, recombinant caspases-3, -6 , or -7 ( $25 \mathrm{ng}$, Sigma) were incubated for $16 \mathrm{~h}$ at $37^{\circ} \mathrm{C}$ with $2 \mu \mathrm{l}$ of reticulocyte lysates in $50 \mu \mathrm{l}$ of $25 \mathrm{mM}$ HEPES (4-(2hydroxyethyl)piperazine-1-ethanesulfonic acid) buffer $\mathrm{pH} 7.5$ containing $0.1 \%$ CHAPS ((3-[(3-cholamidopropyl)dimetylammonio]-1-propanesulfonate)) and $5 \mathrm{mM}$ dithiothreitol. In a subset of experiments, the caspase inhibitor Acetyl-AspartylGlutamyl-Valine-Aspartyl-aldehyde $(10 \mu \mathrm{M})$ was preincubated with caspases before the addition of the reticulocyte lysates. Proteins were then electrophoresed on $12 \%$ PAGE and autoradiographed using Amersham Biosciences hyperfilms. 
Caspase-3 activity measurements. Cells were grown in 6-well plates and incubated without or with STS or 6-hydroxydopamine $(6 \mathrm{OHDA})$ for various time and concentrations. In some cases, cells were pre-treated or not for $30 \mathrm{~min}$ with LY294002 $(10 \mu \mathrm{M})$. Caspase-3-like activity was fluorimetrically measured as extensively detailed. ${ }^{52}$

Real-time quantitative PCR. Total RNA from cells was extracted using the RNeasy kit following the instructions of the manufacturer (Qiagen, Hilden, Germany). After, DNase I treatment, $2 \mu \mathrm{g}$ of total RNA was reverse-transcribed using oligo (dT) priming and avian myeloblastosis virus reverse transcriptase (Promega). Real-time PCR was performed in an ABI PRISM 5700 Sequence Detector System (Applied Biosystems, Foster City, CA, USA), using the SYBR Green detection protocol recommended by the manufacturer. Specific-gene primers were designed by means of the Primer Express software (Applied Biosystems) and are the following: mouse p53-specific primers: forward, $5^{\prime}$-TCCCATCACT TCACTCCTCC-3' and reverse, 5'-AAAAGGCAGCAGAAGGG-3'; V5-specific primers for V5-tagged-DJ-1: forward, $5^{\prime}$-GCGGTTCGAAGGTAAGCCTA-3' and reverse, $5^{\prime}$-GCGGTAGAATCGAGACCGAG-3'; mouse DJ-1-specific primers: forward, $5^{\prime}$-GGAGATGCAAAAACGCAGGG-3' and reverse, 5'-TCCTCCTGG AAG AACCACCA-3'. Relative expressions levels of p53, DJ-1 and V5-tagged-DJ-1 genes were normalized for RNA concentrations with the housekeeping gene mouse $\gamma$-actin using the following primers: forward, $5^{\prime}$-CACCATCGGTTGTTAGTTGCC-3', and reverse, $5^{\prime}$-CAG GTGTCGATGCAAACGTT-3'.

Semi-quantitative PCR analysis of N-Cas, WTC-Cas and 166CCas mRNA. One $\mu \mathrm{g}$ of total RNA obtained as above was used to amplify DJ-1 caspase-derived products by means of Access RT-PCR System kit (Promega) according to manufacturer conditions. The PCRs were performed with either V5-specific primers: forward, $5^{\prime}$-GCGGTTCGAAGGTAAGCCTA- $3^{\prime}$ and reverse, 5'-GCGGTAGAATCGAGACCGAG-3' or GAPDH (control housekeeper gene) forward, 5'-TGGGCTACACTGAGCACCAG-3' and reverse, 5'-CAG-CGTCAAAGGTGGA GGAG- $3^{\prime}$. PCR products were analyzed on a $2 \%$ agarose gel stained with ethidium bromide.

p53 activity, expression and promoter transactivation. The p53 activity was measured by means of the two $p 53$ reporter genes $p 21^{\text {waf }-1}$-luciferase and PG13-luciferase constructs ${ }^{53}$ (provided by Dr B Vogelstein, Baltimore, MD, USA) as previously described. ${ }^{50}$ The transcriptional activation of the human p53 promoter (hpp53) ${ }^{54}$ was measured after transfection of the cDNA coding for the human $\mathrm{p} 53$ promoter sequence in frame with luciferase (provided by Dr M Oren, Rehovot, Israell) as previously described. ${ }^{55}$ All activities were measured after cotransfection of the above cDNAs $(0.5-1 \mu \mathrm{g})$ and $\beta$-galactosidase $(0.25-0.5 \mu \mathrm{g})$ cDNA, to normalize transfection efficiencies (Promega, Madison, WI, USA).

p53 immunoreactivity was analyzed by western blot using an anti-p53 mouse monoclonal antibody (1:5000 dilution) in nuclear extracts prepared as previously described for cytochrome $c$ translocation experiments. ${ }^{50}$

Coimmunoprecipitation experiments. Mouse Embryonic Fibroblasts were lysed in $10 \mathrm{mM}$ Tris- $\mathrm{HCl}$ pH7.5 buffer containing $150 \mathrm{mM} \mathrm{NaCl}, 0.5 \%$ Triton $\mathrm{X}-100,0.5 \%$ deoxycholate, $5 \mathrm{mM}$ EDTA and a protease inhibitors cocktail (Sigma, P2714, according to manufacturer's conditions) gently homogenized and centrifuged at 14000 r.p.m. to remove cellular debris. Coimmunoprecipitations of wild-type and mutated DJ-1 and p53 were performed using ExactaCruz IP/western blot kit following the manufacturer's instructions (Santa Cruz Biotechnology, Santa Cruz, CA, USA). Samples were resolved on 12\% SDS-PAGE and DJ-1 and p53 immunoreactivities were analyzed with the adequate primary (see antibodies and materials section) monoclonal or polyclonal antibodies.

Analysis of DJ-1 and p53 expression in mouse brain tissues. DJ1 and caspase- 6 knockout mice have been recently described. ${ }^{6,56,57}$ All brain samples were homogenized with a Potter apparatus in $10 \mathrm{mM}$ Tris- $\mathrm{HCl}$ pH7.5 buffer complemented with a protease inhibitor cocktail and resolved on $12 \%$ SDS-PAGE gels. DJ-1 and p53 immunoreactivities were detected as described above.

Normal and pathological human brain tissues. All human substantia nigra brain samples were obtained from the GIE Neuro-CEB network (La PitiéSalpêtrière, Paris, France). These samples include four ALS patients (control nonPD-associated pathology): 1811 (male, 55 years old), 1821 (female, 68 years old), 1822 (male, 64 years old), 1823 (female, 62 years old), one sample from an aged matched healthy control patient 3659 (male, 61 years old), and five samples from PD patients: 3605 (male, 64 years old), 4489 (male, 75 years old), 4513 (female, 77 years old), 5193 (male, 75 years old) and 8460 (male, 66 years old). The mean postmortem delay was $21.8 \pm 8.5 \mathrm{~h}$. Samples were homogenized with a Potter apparatus in approximately $300 \mu \mathrm{l}$ of lysis buffer $10 \mathrm{mM}$ Tris- $\mathrm{HCl}$ pH7.5 containing a protease inhibitor cocktail (Sigma). Equal amounts of protein were then resolved on $12 \%$ SDS-PAGE gels and incubated overnight with anti-DJ-1, p53 and caspase-6 antibodies.

Statistical analysis. Statistical analyses were performed with PRISM software (GraphPad Software, San Diego, CA, USA) by using the NewmannKeuls multiple comparison tests for one-way analysis of variance or the unpaired Student's test for pairwise comparisons.

Acknowledgements. We thank $\mathrm{M}$ Oren, and B Vogelstein for providing us p53 promoter and PG13 constructs, respectively. Dr M Roussel is thanked for providing us the $p 19^{\text {arf }-l-}$ and $p 19^{\text {arf }-1-, p 53-l-}$ knockout cells and Drs A Rongvaux and T Town for providing caspase-6 knockout mice brains. We thank Drs S Wilk and $\mathrm{P}$ Morain for providing Benzylocarbonyl-lle-Leu-(OBut)-Ala-leucinal, benzyloxycarbonyl-leucinal and $\mathrm{S} 17092$ inhibitors, respectively. We are grateful to Franck Aguila for his help for artwork. The resource center GIE Neuro-CEB is thanked for providing the human brain samples. This work was supported by the Fondation pour la recherche médicale.

1. Gasser T. Genetics of Parkinson's disease. Curr Opin Neurol 2005; 18: 363-369.

2. Abou-Sleiman PM, Healy DG, Quinn N, Lees AJ, Wood NW. The role of pathogenic DJ-1 mutations in Parkinson's disease. Ann Neurol 2003; 54: 283-286.

3. Hedrich K, Djarmati A, Schafer N, Hering R, Wellenbrock C, Weiss PH et al. DJ-1 (PARK7) mutations are less frequent than Parkin (PARK2) mutations in early-onset Parkinson disease. Neurology 2004; 62: 389-394.

4. Bandopadhyay R, Kingsbury AE, Cookson MR, Reid AR, Evans IM, Hope AD et al. The expression of DJ-1 (PARK7) in normal human CNS and idiopathic Parkinson's disease. Brain 2004; 127 (Part 2): 420-430.

5. Takahashi-Niki K, Niki T, Taira T, Iguchi-Ariga SM, Ariga H. Reduced anti-oxidative stress activities of DJ-1 mutants found in Parkinson's disease patients. Biochem Biophys Res Commun 2004; 320: 389-397.

6. Goldberg MS, Pisani A, Haburcak M, Vortherms TA, Kitada T, Costa C et al. Nigrostriatal dopaminergic deficits and hypokinesia caused by inactivation of the familial Parkinsonismlinked gene DJ-1. Neuron 2005; 45: 489-496.

7. Lee SJ, Kim SJ, Kim IK, Ko J, Jeong CS, Kim GH et al. Crystal structures of human DJ-1 and Escherichia coli Hsp31, which share an evolutionarily conserved domain. J Biol Chem 2003; 278: 44552-44559.

8. Hod Y, Pentyala SN, Whyard TC, El-Maghrabi MR. Identification and characterization of a novel protein that regulates RNA-protein interaction. J Cell Biochem 1999; 72: 435-444.

9. Takahashi K, Taira T, Niki T, Seino C, Iguchi-Ariga SM, Ariga H. DJ-1 positively regulates the androgen receptor by impairing the binding of PIASx alpha to the receptor. J Biol Chem 2001; 276: 37556-37563.

10. Palvimo JJ. PIAS proteins as regulators of small ubiquitin-related modifier (SUMO) modifications and transcription. Biochem Soc Trans 2007; 35 (Part 6): 1405-1408.

11. Shinbo Y, Niki T, Taira T, Ooe H, Takahashi-Niki K, Maita C et al. Proper SUMO-1 conjugation is essential to DJ-1 to exert its full activities. Cell Death Differ 2006; 13: 96-108.

12. Kinumi T, Kimata J, Taira T, Ariga H, Niki E. Cysteine-106 of DJ-1 is the most sensitive cysteine residue to hydrogen peroxide-mediated oxidation in vivo in human umbilical vein endothelial cells. Biochem Biophys Res Commun 2004; 317: 722-728.

13. Canet-Aviles RM, Wilson MA, Miller DW, Ahmad R, McLendon C, Bandyopadhyay S et al. The Parkinson's disease protein DJ-1 is neuroprotective due to cysteine-sulfinic aciddriven mitochondrial localization. Proc Natl Acad Sci USA 2004; 101: 9103-9108.

14. Taira $T$, Saito $Y$, Niki T, Iguchi-Ariga SM, Takahashi $K$, Ariga $H$. DJ-1 has a role in antioxidative stress to prevent cell death. EMBO Rep 2004; 5: 213-218.

15. Moore DJ, Zhang L, Dawson TM, Dawson VL. A missense mutation (L166P) in DJ-1, linked to familial Parkinson's disease, confers reduced protein stability and impairs homooligomerization. J Neurochem 2003; 87: 1558-1567.

16. Blum D, Torch S, Lambeng N, Nissou M-F, Benabid A-L, Sadoul R et al. Molecular pathways involved in the neurotoxicity of 6OH-DOPA and MPTP: contribution to the apoptotic theory in Parkinson's disease. Prog Neurobiol 2001; 65: 135-172.

17. Lazebnik YA, Kaufmann SH, Desnoyers S, Poirier GG, Earnshaw WC. Cleavage of poly(ADP-ribose) polymerase by a proteinase with properties like ICE. Nature 1994; 371: 346-347.

18. Weber JD, Jeffers JR, Rehg JE, Randle DH, Lozano G, Roussel MF et al. p53-independent functions of the p19(ARF) tumor suppressor. Genes Dev 2000; 14: 2358-2365. 
19. Oren M. Regulation of the p53 tumor suppressor protein. J Biol Chem 1999; 274 36031-36034.

20. Brooks $\mathrm{CL}$, Gu W. Ubiquitination, phosphorilation and acetylation: the molecular basis for p53 regulation. Curr Op Cell Biol 2003; 15: 1-8.

21. Mayo LD, Donner DB. A phosphatidylinositol 3-kinase/Akt pathway promotes translocation of Mdm2 from the cytoplasm to the nucleus. Proc Natl Acad Sci USA 2001; 98: 11598-11603.

22. Ogawara $Y$, Kishishita S, Obata T, Ysazawa $Y$, Suzuki T, Tanaka K et al. Akt enhances mdm2-mediated ubiquitination and degradation of p53. J Biol Chem 2002; 277 21843-21850.

23. McCubrey JA, Steelman LS, Chappell WH, Abrams SL, Wong EW, Chang F et al. Roles of the Raf/MEK/ERK pathway in cell growth, malignant transformation and drug resistance. Biochim Biophys Acta 2007; 1773: 1263-1284.

24. Jeong SJ, Pise-Masison CA, Radonovich MF, Park HU, Brady JN. Activated AKT regulates NF-kappaB activation, p53 inhibition and cell survival in HTLV-1-transformed cells. Oncogene 2005; 24: 6719-6728

25. Milne D, Kampanis P, Nicol S, Dias S, Campbell DG, Fuller-Pace F et al. A novel site of AKT-mediated phosphorylation in the human MDM2 onco-protein. FEBS Lett 2004; 577 270-276.

26. Ozes ON, Mayo LD, Gustin JA, Pfeffer SR, Pfeffer LM, Donner DB. NF-kappaB activation by tumour necrosis factor requires the Akt serine-threonine kinase. Nature 1999; 401: 82-85.

27. Moll UM, Riou G, Levine AJ. Two distinct mechanisms alter p53 in breast cancer: mutation and nuclear exclusion. Proc Natl Acad Sci USA 1992; 89: 7262-7266.

28. Shendelman S, Jonason A, Martinat C, Leete T, Abeliovich A. DJ-1 is a redox-dependent molecular chaperone that inhibits alpha-synuclein aggregate formation. PLoS Biol 2004; 2 e362.

29. Xiong Y, Hannon GJ, Zhang H, Casso D, Kobayashi R, Beach D. p21 is a universal inhibitor of cyclin kinases. Nature 1993; 366: 701-704.

30. Miller DW, Ahmad R, Hague S, Baptista MJ, Canet-Aviles R, McLendon C et al. L166P mutant DJ-1, causative for recessive Parkinson's disease, is degraded through the ubiquitin-proteasome system. J Biol Chem 2003; 278: 36588-36595.

31. Villa P, Kaufmann SH, Earnshaw WC. Caspases and caspase inhibitors. Trends Biochem Sci 1997; 22: 388-393.

32. Alves da Costa C. DJ-1 a new comer in Parkinson's disease pathology. Curr Mol Med 2007: 7: 650-657.

33. Jellinger K, Linert L, Kienzl E, Herlinger E, Youdim MB. Chemical evidence for 6-hydroxydopamine to be an endogenous toxic factor in the pathogenesis of Parkinson's disease. J Neural Transm Suppl 1995; 46: 297-314.

34. Bretaud S, Allen C, Ingham PW, Bandmann O. p53-dependent neuronal cell death in a DJ1-deficient zebrafish model of Parkinson's disease. J Neurochem 2007; 100: 1626-1635.

35. Yang Y, Gehrke S, Haque ME, Imai Y, Kosek J, Yang L et al. Inactivation of Drosophila DJ1 leads to impairments of oxidative stress response and phosphatidylinositol 3-kinase/Akt signaling. Proc Natl Acad Sci USA 2005; 102: 13670-13675.

36. Deffie $A, W u H$, Reinke $V$, Lozano $G$. The tumor suppressor $p 53$ regulates its own transcription. Mol Cell Biol 1993; 13: 3415-3423.

37. Engelender S, Kaminsky Z, Guo X, Sharp AH, Amaravi RK, Kleiderlein JJ et al. Synphilin-1 associates with alpha-synuclein and promotes the formation of cytosolic inclusions. Nat Genet 1999; 22: 110-114.

38. Giaime E, Sunyach C, Herrant M, Grosso S, Auberger P, McLean P et al. Caspase 3derived $\mathrm{C}$-terminal product of synphilin-1 displays anti-apoptotic function via modulation of the p53-dependent cell death pathway. J Biol Chem 2006; 281: 11515-11522.
39. Duan W, Zhu X, Ladenheim B, Qian-sheng Y, Guo Z, Oyler J et al. p53 inhibitors preserve dopamine neurons and motor function in experimental parkinsonism. Ann Neurol 2002; 52 597-606.

40. Blum D, Wu Y, Nissou M-F, Arnaud S, Benabid A-L, Verna J-M. p53 and Bax activation in 6-hydroxydopamine-induced apoptosis in PC12 cells. Brain Res 1997; 751: 139-142.

41. Kahns S, Kalai M, Jakobsen LD, Clark BF, Vandenabeele P, Jensen PH. Caspase-1 and caspase-8 cleave and inactivate cellular parkin. I Biol Chem 2003; 278: 23376-23380.

42. Dong Z, Ferger B, Paterna J-C, Vogel D, Furler S, Osinde M et al. Dopamine-dependent neurodegeneration in rats induced by viral vector-mediated overexpression of the parkin target CDC-rel1. Proc Natl Acad Sci USA 2003; 100: 12438-12443.

43. Yang Y, Nishimura I, Imai Y, Takahashi R, Lu B. Parkin suppresses dopaminergic neuronselective neurotoxicity induced by Pael-R in Drosophila. Neuron 2003; 37: 911-924.

44. Henn IH, Gostner JM, Lackner P, Tatzelt J, Winklhofer KF. Pathogenic mutations inactivate parkin by distinct mechanisms. J Neurochem 2005: 92: 114-122.

45. Darios F, Corti O, Lücking CB, Hampe C, Miuriel M-P, Abbas N et al. Parkin prevents mitochondrial swelling and cytochrome $\mathrm{C}$ release in mitochondria dependent cell death. Hum Mol Gen 2003; 12: 517-526.

46. Kamijo T, Zindy F, Roussel MF, Quelle DE, Downing JR, Ashmun RA et al. Tumor suppression at the mouse INK4a locus mediated by the alternative reading frame product p19ARF. Cell 1997; 91: 649-659.

47. Pages G, Guerin S, Grall D, Bonino F, Smith A, Anjuere F et al. Defective thymocyte maturation in p44 MAP kinase (Erk 1) knockout mice. Science 1999; 286: 1374-1377.

48. Sunyach C, Cisse MA, da Costa CA, Vincent B, Checler F. The C-terminal products of cellular prion protein processing, $\mathrm{C} 1$ and $\mathrm{C} 2$, exert distinct influence on p53-dependent staurosporine-induced caspase-3 activation. J Biol Chem 2007; 282: 1956-1963.

49. Alves Da Costa C, Paitel E, Vincent B, Checler F. Alpha-synuclein lowers p53-dependent apoptotic response of neuronal cells. Abolishment by 6 -hydroxydopamine and implication for Parkinson's disease. J Biol Chem 2002; 277: 50980-50984.

50. Alves da Costa C, Dunys J, Brau F, Wilk S, Cappai R, Checler F. 6-Hydroxydopamine but not 1-methyl-4-phenylpyridinium abolishes alpha-synuclein anti-apoptotic phenotype by inhibiting its proteasomal degradation and by promoting its aggregation. J Biol Chem 2006; 281: 9824-9831.

51. Shang $\mathrm{H}$, Lang $\mathrm{D}$, Jean-Marc $B$, Kaelin-Lang A. Localization of DJ-1 mRNA in the mouse brain. Neurosci Lett 2004; 367: 273-277.

52. Alves da Costa C, Ancolio K, Checler F. Wild-type but not Parkinson's disease-related Ala53Thr- $\alpha$-synuclein protect neuronal cells from apoptotic stimuli. J Biol Chem 2000; 275 : 24065-24069.

53. El-Deiry W, Kern S, Pietenpol J, Kinzler K, Vogelstein B. Definition of a consensus binding site for p53. Nat Gen 1992; 1: 45-49.

54. Ginsberg D, Oren M, Yaniv M, Piette J. Protein-binding elements in the promoter region of the mouse p53 gene. Oncogene 1990; 5: 1285-1290.

55. Alves da Costa C, Masliah E, Checler F. $\beta$-synuclein displays an antiapoptotic p53dependent phenotype and protects neurons from 6-hydroxydopamine-induced caspase-3 activation. J Biol Chem 2003; 278: 37330-37335.

56. Le DA, Wu Y, Huang Z, Matsushita K, Plesnila N, Augustinack JC et al. Caspase activation and neuroprotection in caspase-3- deficient mice after in vivo cerebral ischemia and in vitro oxygen glucose deprivation. Proc Natl Acad Sci USA 2002; 99: 15188-15193.

57. Zandy AJ, Lakhani S, Zheng T, Flavell RA, Bassnett S. Role of the executioner caspases during lens development. J Biol Chem 2005; 280: 30263-30272.

\section{Supplementary Information accompanies the paper on Cell Death and Differentiation website (http://www.nature.com/cdd)}

